\title{
Ants of the Monomorium monomorium species-group (Hymenoptera: Formicidae) in the Arabian Peninsula with description of a new species from southwestern Saudi Arabia
}

\author{
Mostafa Sharaf ${ }^{\text {Corresp., }}{ }^{1}$, Hathal M Al Dhafer ${ }^{1}$, Abdulrahman S Aldawood ${ }^{1}$, Francisco Hita Garcia ${ }^{2}$ \\ 1 Department of Plant Protection, College of Food and Agriculture Sciences, King Saud University, Riyadh, Riyadh, Kingdom of Saudi Arabia \\ 2 Okinawa Institute of Science and Technology, Okinawa, Japan \\ Corresponding Author: Mostafa Sharaf \\ Email address: mosharaf@ksu.edu.sa
}

We revise the taxonomy of the myrmicine ants of the Monomorium monomorium speciesgroup for the Arabian Peninsula. Six species are recognized: Monomorium aeyade Collingwood \& Agosti, 1996, M. clavicorne André, 1881, M. exiguum Forel, 1894, M. holothir Bolton, 1987, M. mohammedi sp. n., and M. sarawatense Aldawood \& Sharaf, 2013. On the basis of the worker caste, we describe Monomorium mohammedi sp. n. from the southwestern region of the Kingdom of Saudi Arabia (KSA). We designate a neotype for Monomorium aeyade Collingwood \& Agosti and redescribe and illustrate the worker caste. Furthermore, we provide a worker-based species identification key, distribution maps for the treated species, and ecological and biological notes, if available. Monomorium holothir is recorded for the first time from the KSA. Also, we propose M. clavicorne var. punica Santschi, 1915 as junior synonym of M. clavicorne, as well as M. dryhimi Aldawood \& Sharaf, 2011 and M. montanum Collingwood \& Agosti, 1996 be treated as junior synonyms of Monomorium exiguum. 
1 Ants of the Monomorium monomorium species-group (Hymenoptera: Formicidae) in the 2 Arabian Peninsula with description of a new species from southwestern Saudi Arabia

3 Mostafa R. Sharaf ${ }^{1 *}$, Hathal M. Al Dhafer ${ }^{1}$, Abdulrahman S. Aldawood ${ }^{1}$, Francisco Hita Garcia ${ }^{2}$

41 Department of Plant Protection, College of Food and Agriculture Sciences, King Saud

5 University, Riyadh, Kingdom of Saudi Arabia.

62 Okinawa Institute of Science and Technology, Onna-son, Okinawa, Japan.

7 *Author to whom correspondence should be addressed e-mail: mosharaf@ksu.edu.sa

ABSTRACT

9 We revise the taxonomy of the myrmicine ants of the Monomorium monomorium species-group for the Arabian Peninsula. Six species are recognized: Monomorium aeyade Collingwood \& Agosti, 1996, M. clavicorne André, 1881, M. exiguum Forel, 1894, M. holothir Bolton, 1987, M. mohammedi sp. n., and M. sarawatense Aldawood \& Sharaf, 2013. On the basis of the worker caste, we describe Monomorium mohammedi sp. n. from the southwestern region of the Kingdom of Saudi Arabia (KSA). We designate a neotype for Monomorium aeyade Collingwood \& Agosti and redescribe and illustrate the worker caste. Furthermore, we provide a worker-based species identification key, distribution maps for the treated species, and ecological and biological notes, if available. Monomorium holothir is recorded for the first time from the KSA. Also, we propose M. clavicorne var. punica Santschi, 1915 as junior synonym of M. clavicorne, as well as M. dryhimi Aldawood \& Sharaf, 2011 and M. montanum Collingwood \& Agosti, 1996 be treated as junior synonyms of Monomorium exiguum.

\section{INTRODUCTION}

The ant genus Monomorium is one of the most diverse genera in the subfamily Myrmicinae with 388 described species and subspecies (Bolton, 2017). It is distributed worldwide throughout all zoogeographic regions, with most species occurring in the Old World tropics and temperate zones (Brown, 2000). Considering how diverse and widespread the genus is, very little information on the natural history of most species exists, especially for the M. monomorium species-group (Bolton, 1987). Apparently, most species inhabit the topsoil layer or leaf litter and seem to have a rather generalist diet. The taxonomic foundation for the genus as a whole is in a moderate state based on some regional revisions (e.g. Bolton, 1987; Heterick, 2001, 2006), as well as faunistic treatments providing local or regional keys (e.g. Collingwood \& Agosti, 1996;

31 Terayama, 2009; Sarnat \& Economo, 2012). The available revisionary contributions on the fauna 
32 of the M. monomorium species-group are scarce, and basically consist of Bolton (1987) for the

33 Afrotropical fauna and Heterick $(2001,2006)$ for the Australian and Malagasy faunas

34 respectively. However, these include many species now removed from the genus.

35 The taxonomic history of the M. monomorium species-group in the Arabian Peninsula is a set of

36 single distribution records and single species descriptions scattered through the literature. The

37 first published work on the Arabian Monomorium fauna (Collingwood, 1985) recorded a couple

38 of species of the M. monomorium species-group from the Kingdom of Saudi Arabia (KSA),

39 namely M. clavicorne Andre, 1881 and M. montanum Collingwood \& Agosti, 1996 (initially

40 misidentified as M. zulu Santschi, 1914 in Collingwood, 1985). In a later faunistic contribution

41 Collingwood \& Agosti (1996) listed eight species, and described five new species, M. aeyade

42 Collingwood \& Agosti, 1996, M. baushare Collingwood \& Agosti, 1996, M. desertorum

43 Collingwood \& Agosti, 1996, M. montanum Collingwood \& Agosti, 1996, and M. qarahe

44 Collingwood \& Agosti, 1996.

45

46 The first record of the species M. exiguum Forel was published by Aldawood \& Sharaf (2009)

47 from the Asir Mountains (KSA). Aldawood \& Sharaf (2011) described M. dryhimi, based on the

48 worker caste from the southwestern Mountains of the KSA, and provided a key to the Arabian

49 species. El-Hawagry et al. (2013) described M. sarawatensis Sharaf \& Aldawood from the Al

Baha Province based on the worker caste, and provided a key to the Arabian species of the $M$.

monomorium species-group. Recently, M. desertorum Collingwood \& Agosti, 1996 was

synonymized with M. exiguum Forel, 1894 (Sharaf et al., 2015), and Sharaf et al. (2017)

produced a key to the Monomorium fauna of the Socotra Archipelago, described M. elghazalyi

Sharaf \& Aldawood, 2017, and synonymized M. baushare and M. qarahe under M. exiguum.

In the Malagasy region, M. exiguum is the most abundant species in leaf litter samples (Heterick, 2006). However, this group of ants is taxonomically difficult due to lack of revisionary work, in addition to the small body size and pale body colors that make the ants frequently overlooked by collectors and consequently poorly represented in the regional museums and collections. Ants of the M. monomorium-group are among the most abundant Monomorium in the Arabian Peninsula, commonly represented in ecological and biodiversity research projects of the region.

In this study, we provide the first comprehensive revision of the M. monomorium species- 
63 the KSA and re-describe the five previously known species. For all species we present detailed 64 descriptions, diagnoses, high-quality montage images, and distribution maps. Furthermore, we provide a new illustrated identification key to the species of the species group on the basis of the 66 worker caste.

\section{MATERIAL AND METHODS}

The species names follow the online catalogue of ants of the world (Bolton, 2017). Distribution maps were made using DIVA-GIS (version 7.5.0.0). Digital color images of lateral and dorsal views of the entire body and full-face views of the head of each species were created using a Leica DFC450 digital camera with a Leica Z16 APO microscope and LAS (v3.8) software. These images are also available online on AntWeb (www.AntWeb.org) and are accessible through unique specimen identifiers attached (e.g. CASENT0922329). Throughout the text, 'w' stands for 'worker' or 'workers'.

\section{Institutional abbreviations}

The collection abbreviations follow Evenhuis (2009) and Brandão (2000).

Measurements and indices Figures 1, 2, 3:

All measurements are in millimeters and follow the standard measurements of previous works on the genus (Bolton, 1987; Sharaf et al., 2017):

$91 \mathrm{EL}=$ Eye Length; maximum diameter of eye in lateral view.

$92 \mathrm{EM}=$ Distance between anterior margin of eye and mandibular insertion in lateral view.

$93 \mathrm{HL}=$ Head Length; maximum length of head, excluding mandibles in full-face view. 
$94 \mathrm{HW}=$ Head Width; maximum width of head behind eyes in full-face view.

$95 \mathrm{ML}=$ Mesosoma Length (=Weber Length); length of mesosoma in lateral view; from a point at 96 which pronotum meets cervical shield to posterior base of propodeal lobes or teeth.

$97 \mathrm{PPH}=$ Postpetiole Height; maximum height measured in lateral view.

$98 \mathrm{PPL}=$ Postpetiole Length; maximum length measured in dorsal view.

99 PPW $=$ Postpetiole Width; maximum width measured in dorsal view.

$100 \mathrm{PTH}=$ Petiole Height; maximum height measured in lateral view.

$101 \mathrm{PTL}=$ Petiole Length; maximum length measured in dorsal view, from anterior margin to 102 posterior margin.

103 PTW $=$ Petiole Width; maximum width measured in dorsal view.

$104 \mathrm{PW}=$ Pronotal Width; maximum width in dorsal view.

$105 \mathrm{SL}=$ Scape Length, excluding basal neck.

$106 \mathrm{TL}=$ Total Length, sum of lengths of head, mesosoma, petiole, postpetiole and gaster in 107 profile.

108

109 Indices

$110 \mathrm{CI}=$ Cephalic Index $(\mathrm{HW} / \mathrm{HL} \times 100)$.

$111 \mathrm{EI}=$ Eye Index $(\mathrm{EL} / \mathrm{HW} \times 100)$.

$112 \mathrm{SI}=\quad$ Scape Index $(\mathrm{SL} / \mathrm{HW} \times 100)$.

\section{Nomenclatural acts}

114 The electronic version of this article in Portable Document Format (PDF) will represent a

115 published work according to the International Commission on Zoological Nomenclature (ICZN),

116 and hence the new names contained in the electronic version are effectively published under that

117 Code from the electronic edition alone. This published work and the nomenclatural acts it

118 contains have been registered in ZooBank, the online registration system for the ICZN. The

119 ZooBank LSIDs (Life Science Identifiers) can be resolved and the associated information viewed

120 through any standard web browser by appending the LSID to the prefix http://zoobank.org/. The

121 LSID for this publication is: urn:Isid:zoobank.org:pub:58913E59-AEE4-43BD-881E-

$122 \mathrm{C} 23 \mathrm{CD} 61 \mathrm{~F} 44 \mathrm{~F} 9$. The online version of this work is archived and available from the following 123 digital repositories: PeerJ, PubMed Central and CLOCKSS. 
125 Diagnosis of Arabian ants in the Monomorium monomorium species-group Within the genus

126 Monomorium, workers of the M. monomorium species-group can be easily recognized by the

127 following combination of characters (Bolton, 1987): monomorphic, with size variation; median

128 clypeal portion raised, projecting anteriorly and longitudinally bicarinate; anterior clypeal margin

129 without a pair of teeth; dorsal surface of mandibles unsculptured and masticatory margin armed

130 with four teeth, decreasing in size from apex to base; antennae with 10 to 12 segments,

131 terminating in a well-defined three-segmented club; eyes present but variable in size, situated in

132 front of the midlength of the sides in full-face view, and with four or more ommatidia in the

133 longest row; head longer than broad; cephalic dorsum smooth and shining; metanotal groove

134 impressed, with distinct cross-ribs; propodeal spiracle circular to subcircular; propodeal dorsum

135 meeting declivity in a rounded angle; promesonotum and propodeal dorsum unsculptured; body

136 pilosity variable in distribution but usually conspicuous, rarely absent from mesosomal dorsum;

137 petiole, postpetiole and gastral tergites usually unsculptured.

138

139 Synoptic species list and distribution of Arabian ants in the Monomorium monomorium

140 species-groupMonomorium aeyade Collingwood \& Agosti, 1996

141 Monomorium clavicorne André, 1881

142 = Monomorium clavicorne punicum Santschi, 1915 syn. nov.

143 Monomorium exiguum Forel, 1894

$144=$ Monomorium exiguum var. bulawayensis Forel, 1913b

$145=$ Monomorium faurei Santschi, 1915b

$146=$ Monomorium exiguum r. flavescens Forel, 1916

$147=$ Monomorium montanum Collingwood \& Agosti, 1996 syn. nov.

$148=$ Monomorium dryhimi Aldawood \& Sharaf, 2011 syn. nov.

149 Monomorium holothir Bolton, 1987

150 Monomorium mohammedi Sharaf \& Hita Garcia sp. n.

151 Monomorium sarawatense Aldawood \& Sharaf, 2013

152 Monomorium. exiguum Forel, 1894 is the most widespread species of the M. monomorium

153 species-group of the Arabian Peninsula. It invades a broad range of habitats in the region

154 whereas the remaining five species, M. aeyade Collingwood \& Agosti, 1996, M. clavicorne

155 André, 1881, M. holothir Bolton, 1987, M. mohammedi sp. n., and M. sarawatense Aldawood \& 
156 Sharaf, 2013 are more restricted in distribution Figure 4. Further distribution information will be 157 presented under each species.

158 Identification Key to the Arabian species of the Monomorium monomorium-group

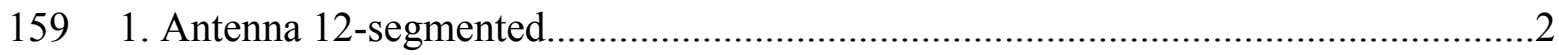

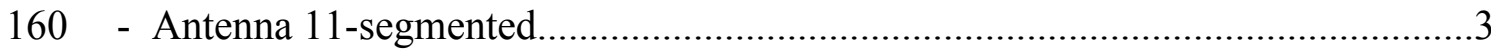

161 2. Body pilosity clubbed; mesosoma, petiole and postpetiole distinctly sculptured

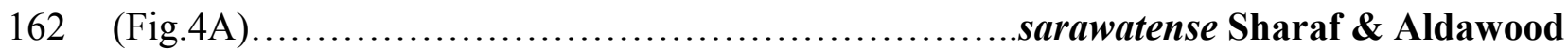

163 - Body pilosity simple; mesosoma, petiole and postpetiole smooth and shining

164 (Fig.4B) holothir Bolton

165 3. Mesosoma without standing hairs (Figs. 4C, 4D). .4

166 - Mesosoma with standing hairs (Figs. 4E, 4F) .5

167 4. Eyes appearing larger, with a ring of seven to eight ommatidia encircling a single row of 2

168 ommatidia, and in profile closer to mandibular insertions (EM 0.05); meso-and metapleuron

169 smooth; petiole and postpetiole smooth and each with one pair of standing hairs (Fig. 4C)

170 aeyade Collingwood \& Agosti

171 - Eyes appearing smaller, with only 5-6 ommatidia, and in profile further away from mandibular

172 insertions (EM 0.09-0.11); meso-and metapleuron finely shagreened; petiole and postpetiole

173 superficially shagreened and without standing hairs (Fig. 4D) mohammedi sp. n.

174 5. Mesosoma with only two pairs of standing hairs, one on pronotal corners and one propodeum

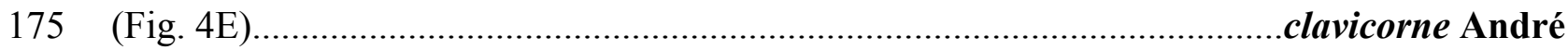

176 - Mesosoma with several pairs of standing hairs, about 10 pairs (Figs. 4F).....exiguum Forel

177 Review of species

178 Monomorium aeyade Collingwood \& Agosti, 1996, Figure 5

179 Monomorium aeyade Collingwood \& Agosti, 1996: 341 (w.)

180 Type material examined

181 Neotype pinned worker: Oman, wadi Aeyad, 20.iii.1990, (M. D. Gallagher) (WMLC:

182 CASENT0922329).

183 Measurements

184 Paratype worker.

185 TL 1.42; HL 0.40; HW 0.31; SL 0.27; EL 0.06; ML 0.40; PW 0.18; PTW 0.07; PTH 0.12; PPW 186 0.09; PPH 0.09; CI 78; EI 19; SI 87. 


\section{Description}

188 Worker. Head. In full-face view distinctly longer than broad with nearly parallel sides and

189 feebly concave posterior margin; median clypeal portion without carina or anterolateral angles,

190 anterior clypeal margin straight or feebly concave; antenna 11-segmented; scapes relatively long

191 (SI 81), when laid straight back from their insertions reach three quarter of head length; eyes

192 oval, small, (EL $0.16 \times \mathrm{HW}$ ) with a ring of ommatidia encircling a single row of 2 ommatidia;

193 frontal lobes farther apart in full-face view. Mesosoma. In profile with flat promesonotal

194 dorsum, which slopes posteriorly to a well-defined metanotal groove; propodeal spiracles small

195 and pinhole-like; propodeal dorsum evenly sloping posteriorly to short declivity. Petiole: Node

196 massive, rounded dorsally, and little higher than postpetiolar node in profile; anterior peduncle

197 short. Postpetiole. Node low and convex dorsally. Sculpture. Entire body surfaces smooth and

198 shining except for the distinct metanotal cross ribs. Pilosity. Underside of head without hairs;

199 cephalic surface with scattered minute hair-pits; anterior clypeal margin and mandibles with

200 longer hairs; antennae with abundant appressed pubescence; mesosoma without hairs; postpetiole

201 two pairs of backward directed hairs; gaster with few longer hairs. Color. Uniform light yellow.

202 Note

203 This species was originally described based on two worker specimens, the holotype and one

204 paratype. During an extensive search in the WMLC collection it was not possible to locate the

205 holotype, which is presumably lost. However, the paratype specimen was available for

206 examination. The original description given by Collingwood \& Agosti (1996) was brief and the

207 diagnostic differentiation was unclear, therefore, we designate a neotype to unequivocally

208 ascertain the identity of the species and re-describe the species.

209 Biological and Ecological notes

210 Nothing is known of the biology or ecology of the species.

211 Geographic range

212 Monomorium aeyade is only known from the type locality in Oman (Table 1).

213 Monomorium clavicorne André, 1881, Figure 6

214 Monomorium clavicorne André, 1881: 68, pl. 3, fig. 9 (w.). [Combination in Monomorium

215 (Lampromyrmex): Wheeler, 1922: 876. Subspecies of Monomorium orientale: Mayr, 1904: 4;

216 Emery, 1908: 685. Raised to species by Santschi, 1915a: 58; Emery, 1922: 183. Current

217 subspecies: nominal plus Monomorium clavicorne punicum.] 
218 Monomorium clavicorne var. punica Santschi, 1915 58, fig. 4 (w.). Syn. nov.

219 Type material examined

220 Of M. clavicorne: Holotype, pinned worker, ISRAEL, Jaffa (MNHN: CASENT0915416).

221 [image examined]

222 Of M. clavicorne punicum: Holotype, pinned worker, TUNISIA: Sousse, 31.XII.1915

223 (Normand) (NHMB: CASENT0913568). [image examined]

224 [Note: Based on the examination of the type images of both, M. clavicorne and M. clavicorne

225 punicum, we propose to synonymize the latter under the first on the basis of morphological

226 similarity and biogeographical considerations].

227 Non-type material examined

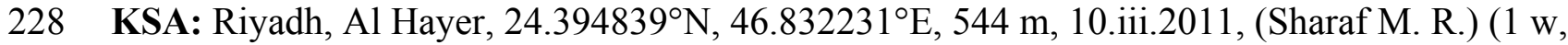
229 KSMA).

230 Previous records. Riyadh, Riyadh Agricultural Centre, $24.501389^{\circ} \mathrm{N}, 46.626111^{\circ} \mathrm{E}$

231 22.iii.83, 19.iv.83; Fayfa, $17.28797^{\circ} \mathrm{N}, 43.14434^{\circ} \mathrm{E}, 27 . i i i .83$; Al Qatif, $26.510278^{\circ} \mathrm{N}$,

232 49.968889 ${ }^{\circ}$ E, 15.iv.83; Hair valley, 17.iv.83 (Collingwood C. A.) (Collingwood 1985).

233 Measurements

234 Worker ( $\mathrm{n}=2)$. TL 1.44-1.48; HL 0.37-0.38; HW 0.28-0.29; SL 0.22-0.25; EL 0.05-0.06; ML

235 0.41-0.42; PW 0.17-0.18; PTL 0.07-0.08; PTW 0.07; PTH 0.09-0.12; PPL 0.05-0.07; PPW

236 0.08; PPH 0.07-0.08; CI 76; EI 17-21; SI 79-86.

237 Description

238 Worker. Head. In full-face view distinctly longer than broad with nearly parallel sides and

239 feebly concave posterior margin; median clypeal portion without carina or anterolateral angles,

240 anterior clypeal margin feebly concave; antenna 11-segmented; terminal funicular segment

241 enlarged, more than twice longer than the two preceding segments; scapes long (SI 79-86); eyes

242 oval, small, (EL $0.17-0.21 \times \mathrm{HW}$ ) with a ring of ommatidia encircling two inner short rows of 2-

2433 ommatidia; frontal lobes farther apart in full-face view. Mesosoma. In profile with a feebly

244 convex promesonotal dorsum, which slopes posteriorly to a well-defined metanotal groove;

245 propodeal spiracles small and pinhole-like; propodeal dorsum evenly sloping posteriorly to short

246 declivity. Petiole: Node massive, narrowly rounded above, and slightly higher than postpetiolar

247 node in profile, anterior peduncle short. Postpetiole. Node small and convex dorsally.

248 Sculpture. Cephalic surface smooth and shining; mandibles smooth and shining, with faint 
249 striations; mesosoma, petiole, postpetiole, and gaster smooth and shining; metanotal cross ribs

250 distinct. Pilosity. Cephalic surface with scattered minute hair-pits; anterior clypeal margin and

251 mandibles with longer hairs; antennae with abundant appressed pubescence; pronotal angles with

252 a pair of long hairs; propodeal dorsum with one pair of hairs; petiole and postpetiole each with

253 one pair of backward directed hairs; gaster with few longer hairs on distal half. Color. Overall

254 uniform clear yellow.

\section{Biological and Ecological notes}

256 Little is known of the biology of the species. The single specimen available was found in a

257 cultivated area with a sewage water stream. It was coexisting with Tapinoma simrothi Krausse,

258 1911, Trichomyrmex mayri (Forel, 1902), and Tetramorium caespitum (Linnaeus, 1758). Due to

259 the relatively broad regional distribution of the species it might be an introduction to the KSA.

260 Geographic range

261 This species was described from Palestine (André, 1881) and recorded from many countries in

262 the Middle East including Iran, Lebanon, Sudan, Syria, Turkey, the KSA (Collingwood, 1985;

263 Collingwood \& Agosti, 1996), the UAE (Collingwood et al., 2011), and several North African

264 countries including Egypt (Sharaf, 2006), Morocco and Tunisia (Table 1).

\section{Monomorium exiguum Forel, 1894, Figure 7}

266 Monomorium exiguum Forel, 1894: 85. [Combination in Monomorium (Martia): Forel, 1913a:

267 351; in Monomorium (Lampromyrmex): Wheeler, 1922: 876.]

268 Monomorium exiguum var. bulawayensis Forel, 1913: 217 (w.) [Combination in Monomorium

269 (Lampromyrmex): Wheeler, 1922: 876. Junior synonym of Monomorium exiguum: Bolton, 1987:

270 388; here confirmed.]

271 Monomorium faurei Santschi, 1915b: 260, fig. 10 (w.). [Combination in Monomorium

272 (Lampromyrmex): Wheeler, 1922: 876. Junior synonym of Monomorium exiguum: Bolton, 1987:

273 388; here confirmed.]

274 Monomorium exiguum r. flavescens Forel, 1916: 418 (w.). [Junior synonym of Monomorium

275 exiguum: Bolton, 1987: 388; here confirmed.]

276 Monomorium minutissimum Santschi, 1937: 227, figs. 27, 28 (w.). [Junior synonym of

277 Monomorium mictile: Bolton, 1987: 401. Junior synonym of Monomorium exiguum: Heterick,

278 2006: 116; here confirmed.] 
279 Monomorium baushare Collingwood \& Agosti, 1996: 342 (w.). [Junior synonym of

280 Monomorium exiguum: Sharaf et al., 2017: 343; here confirmed.]

281 Monomorium desertorum Collingwood \& Agosti, 1996: 344 (w.). [Junior synonym of

282 Monomorium exiguum: Sharaf et al., 2015: 52; here confirmed.]

283 Monomorium montanum Collingwood \& Agosti, 1996: 350, fig. 24 (w.). Syn. nov.

284 Monomorium qarahe Collingwood \& Agosti, 1996: 353 (w.). [Junior synonym of Monomorium

285 exiguum: Sharaf et al., 2017: 343; here confirmed.]

286 Monomorium dryhimi Aldawood \& Sharaf, 2011: 49, figs. 1-7 (w.). Syn. nov.

287 Type material examined

288 Of M. exiguum: Lectotype, pinned worker, ETHIOPIA: Shoa, 3 (MHNG: CASENT0101870).

289 Paralectotype, Shoa, 3 (MHNG: CASENT0101853). [Images of both examined]

290 Of M. baushare: Holotype, pinned worker, OMAN: Sad Baushar, 23.55, 58.4, 2.I.1992

291 (Gallagher, M.D.) (WMLC: CASENT0906342). [examined]

292 Of M. bulawayensis: Lectotype, pinned worker, ZIMBABWE: Bulawayo, (Arnold, G.)

293 (MHNG: CASENT0010763). Paralectotype, pinned worker with same data as lectotype

294 (MHNG: CASENT0010762). [Images of both examined]

295 Of M. dryhimi: Holotype, pinned worker, KSA: Al Bahah province, Amadan forest, Al Mandaq

296 governorate, 20.7496, 41.24743, 1881 m, 19.V.2010 (Sharaf, M. R. \& Aldawood, A. S.)

297 (KSMA). Paratypes, 15 pinned workers with same data as holotype (CASC: CASENT0217367;

298 KSMA; WMLC: CASENT0922344).

299 Of M. faurei: Lectotype, pinned worker, GABON: 31.XII.1914 (Faure, F.) (NHMB:

300 CASENT0010878). Paralectotype, pinned worker with same data as lectotype (NHMB:

301 CASENT0010879). [Images of both examined]

302 Of M. flavescens: Lectotype, pinned worker, DEMOCRATIC REPUBLIC OF CONGO: St.

303 Gabriel, Stanleyville (Kohl) (MHNG: CASENT0101592). Paralectotype, pinned worker with

304 same data as lectotype (MHNG: CASENT0101586). [Images of both examined]

305 Of M. minutissimum: Holotype, pinned worker, Angola, Chemin d' Ebanga (Monard, A.)

306 (NHMN: CASENT0010880). [Images examined]

307 Of M. montanum: Holotype, pinned worker, KSA: Sawdah Mt., 2500 m, 9.IV.1983

308 (Collingwood, C. A.) (WMLC). Paratypes, 4 pinned workers: $1 \mathrm{w}$ same data as holotype

309 (NHMB: CASENT0913825); 1 w Wadi Azizah, 18.IX.1983 (Collingwood, C. A.) (WMLC: 
310 CASENT0906347); $1 \mathrm{w}$ Bishah, 7.IV.1983 (Collingwood, C. A.) (WMLC: CASENT0906345);

$3111 \mathrm{w}$ An-Naamah, 8.IV.1983 (Collingwood, C. A.) (WMLC: CASENT0906346). [examined]

312 Of M. qarahe: Holotype, pinned worker, KSA: Qaraah village, 18.05, 42.75, $2000 \mathrm{~m}$,

313 16.IV.1976 (Büttiker, W.) (WMLC: CASENT0906344). Paratype, one pinned worker with same

314 data as holotype (WMLC). [examined]

315 Non-type material examined

316 CAMEROON: Nkoemvon, 1980, (Jackson D.), (12 w); Nkoemvon, 18.v.1980, (Jackson D.), (6

317 w); Nkoemvon, 04.iii.1980, (Jackson D.), (9 w); Mondoni, 14.iii.1990, (Dejean A.), (6 w);

318 Yaounde, 26.iii.1989, (Dejean A.), (3 w); Mbalmayo, xi.1993, (Stork N.), (3 w); GHANA: Old

319 Tafo nr. Tafo, 31.i.1992, (Belshaw B.), (2 w, 1 q, leaf litter, primary forest); Maabari nr. Tepa,

320 18.xii.1991, (Belshaw B.), (3 w, leaf litter, secondary forest); Asiakwa nr. Kibi, 01.v.1992,

321 (Belshaw B.), (3 w, leaf litter Cocoa); Kibi, 24.iii.1970, (Leston D.), (17 w, litter samples);

322 Manpong, 03.ii.1970, (Room R.), (5 w); Boku, 14.viii.1968, (Collingwood C. A.), (1 w, on

323 cocoa); Pankese, 30.ix.1968, (Collingwood C. A.), (1 w, on cocoa); Mampong, 26.i.1970, (Room

324 P.), (1 w, 1 q); Legon, A. D., 08.vii.1970, (Leston D.), (7 w, 2 q); Tafo, 25.ix.1970, (Bolton B.),

325 (6 w, twig on forest floor); Tafo, Cocoa Research Institution, 23.xii.1991, (Belshaw R.), (3 w,

326 leaf litter, secondary forest); Tafo, 26.ii.1970, (Bolton B.), (2 w, 1 q, log mould); Tafo,

327 26.ii.1970, (Bolton B.), (3 w, log mould samples); Legon, 24.vii.1970, (Leston D.), (3 w);

328 Legon, A. D., 08.x.1970, (Leston D.), (5 w); Legon, A. D., 14.x.1970, (Leston D.), (6 w, 2q);

329 Legon, A. D., 24.vii.1970, (Leston D.), (3 w); Tumu, 25.xii.1969, (Room R.), (2 w); KENYA:

330 Tana R., Kora, 11.vii.1983, (Collins N. M., Ritchie M.), (6 w, pitfall); MADAGASCAR: 48 km

331 ENE, Morondava, 20.066667 $\mathrm{S}, 44.65^{\circ} \mathrm{E}, 07.1 .1991,30 \mathrm{~m}$, (Olson D. M.), (2 w, tropical dry

332 forest, CN6T); NIGERIA: Black pod project, 05.ii.1975, (Taylor B.) (3 w, 1 q); Gambari,

333 16.vi.1969, (Bolton B.), (9 w, on cocoa); Gambari, 20.v.1969, (Bolton B.), (7 w, rotten log);

334 Ibadan, 11.x.1987, (Noyes J.), (21 w), previous material in BMNH; OMAN: Jebel Akhdar,

335 Alain, $23.07279^{\circ} \mathrm{N}, 57.66179^{\circ} \mathrm{E}, 1949 \mathrm{~m}, 4.1 \mathrm{v} .2016$, (Sharaf M. R.) (17 w, KSMA); Jebel

336 Akhdar, Alain, 23.07237N, 57.66187E, 1889 m, 6.iv.2016, (Sharaf M. R.) (4 w, KSMA);

337 Muscat, $23.62405^{\circ} \mathrm{N}, 58.48891^{\circ} \mathrm{E}, 9 \mathrm{~m}, 9.1 \mathrm{v} .2016$, (Sharaf M. R.) (6 w, KSMA); Qurayat,

$33823.20460^{\circ} \mathrm{N}, 58.96920^{\circ} \mathrm{E}, 39 \mathrm{~m}, 8 . \mathrm{iv} .2016$, (Sharaf M. R.) (9 w, KSMA); Date palm, no specific

339 locality, 8.iv.2016, (A. Polaszek) (1 w, KSMA); Nakhl, 23.49327º N, 57.83421 ${ }^{\circ} \mathrm{E}, 190 \mathrm{~m}$,

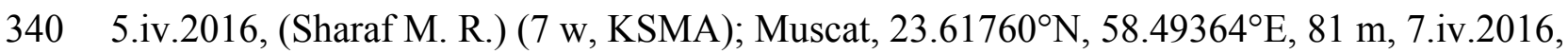


341 (Sharaf M. R.) (1 w, KSMA); W. Fanga, 23.46194ºN, 58.10326 E, 160 m, 20.i.2017, (Sharaf M.

342 R.) (3 w, KSMA); Eastern Hajar, Mts, S. side, Samail gap, Lizurgh village, $23.355556^{\circ}$ N,

$34358.105556^{\circ} \mathrm{E}, 280$ m, 6.iv.2016, (A. Polaszek) (3 w, KSMA); Muscat, KOM, Alraha village,

$34423.56665^{\circ} \mathrm{N}, 58.17630^{\circ} \mathrm{E}, 74 \mathrm{~m}$, (Sharaf M. R.) (1 w, KSMA); Masfat Elebryein, 23.14178 $\mathrm{N}$,

345 57.31330, 933 m, 21.i.2017, (Sharaf M. R.) (3 w, KSMA); Alkhoud village, $23.57154^{\circ} \mathrm{N}$,

$34658.12166^{\circ} \mathrm{E}, 63 \mathrm{~m}$, (Sharaf M. R.) (1 w, KSMA); W. Fanga, $23.45336^{\circ} \mathrm{N}, 58.11807^{\circ} \mathrm{E}, 166 \mathrm{~m}$,

347 20.i.2017, (Sharaf M. R.) (4 w, KSMA); Eastern Hajar, Mnt., Rd to Sur, $23.1675^{\circ}$,

348 58.101944르, 280 m, (A. Polaszek) (2 w, KSMA); Western Hajar Mnt., Nakham, 23.388056² N,

$34957.832222^{\circ}$ E, 310 m, 2.iv.2016, (A. Polaszek) (12 w, KSMA); Hajar Mnt., Wadi Alkhoud,

$35023.566667^{\circ} \mathrm{N}, 58.116667^{\circ} \mathrm{E}, 80 \mathrm{~m}, 13 . \mathrm{i} .2017$, (A. Polaszek) (1 w, KSMA); Batinah coast, Hibra

351 village, $23.493733^{\circ} \mathrm{N}, 57.8337^{\circ} \mathrm{E}, 190 \mathrm{~m}$, 5.iv.2016, (A. Polaszek) (32 w, KSMA); KSA: Al

352 Baha Province: Elqamh park, Baljurshi, $19.913056^{\circ} \mathrm{N}, 41.905^{\circ} \mathrm{E}, 1931 \mathrm{~m}, \mathrm{v} .2010$, (49 w);

353 Elqamh park, Baljurshi, $19.805722^{\circ} \mathrm{N}, 41.711889^{\circ} \mathrm{E}, 1950 \mathrm{~m}$, ix.2011, (39 w); Amadan,

354 Almandaq, $20.245278^{\circ} \mathrm{N}, 41.468333^{\circ} \mathrm{E}, 1881 \mathrm{~m}, \mathrm{v} .2010,(21 \mathrm{w})$; W. Turabah, Almandaq,

$35520.211028^{\circ} \mathrm{N}, 41.288222^{\circ} \mathrm{E}, 1793 \mathrm{~m}, \mathrm{v} .2011$ (13 w); W. Turabah, Almandaq, $20.241917^{\circ} \mathrm{N}$,

$35641.262833^{\circ} \mathrm{E}, 1751 \mathrm{~m}$, ix.2011, (6 w); W. Turabah, Almandaq, $20.211028^{\circ} \mathrm{N}, 41.288222^{\circ} \mathrm{E}$,

3571793 m, v.2011, (30 w); W. Gonouna, AlUrdiya gov., $19.429361^{\circ} \mathrm{N}, 41.605028^{\circ} \mathrm{E}, 353 \mathrm{~m}$,

358 v.2011, (17 w); Al majardah, W. Khat, $19.08913^{\circ} \mathrm{N}, 41.97126^{\circ} \mathrm{E}, 513 \mathrm{~m}$, xi.2012, (1 w); $\mathrm{Al}$

359 Mukhwah, Dhi Ayn, 19.929417º , 41.441722E, 741 m, v.2011, (4 w); Al Mukhwah, Dhi Ayn,

$36019.93143^{\circ} \mathrm{N}, 41.44185^{\circ} \mathrm{E}, 728 \mathrm{~m}$, iv.2016, (10 w, 2 q); Al Mukhwah, Dhi Ayn, $19.92967^{\circ} \mathrm{N}$,

$36141.44291^{\circ} \mathrm{E}, 706 \mathrm{~m}$, iv.2016, (2 w); Al Mukhwah, Dhi Ayn, 19.929417º $, 41.441722^{\circ} \mathrm{E}, 741 \mathrm{~m}$,

362 v.2010, (25 w); Al Mukhwah, Dhi Ayn, 19.92967º N, 41.44291E, 706 m, ix.2011, (3 w); Al

363 Mukhwah, Dhi Ayn, 19.92967ºN, 41.44291E, 744 m, ix.2011, (11 w); Al Mukhwah, Dhi Ayn,

$36419.92967^{\circ} \mathrm{N}, 41.44291^{\circ} \mathrm{E}, 741 \mathrm{~m}, \mathrm{v} .2011$, (3 w); Shada Al A'la, $19.845167^{\circ} \mathrm{N}, 41.30445^{\circ} \mathrm{E}, 741$

365 m, i.2015, (1 w); Shada Al A’la, $19.842917^{\circ} \mathrm{N}, 41.311517^{\circ} \mathrm{E}, 1666 \mathrm{~m}$, xi.2015, (1 w); W.

366 Elzaraeb, $20.073417^{\circ} \mathrm{N}, 41.38675^{\circ} \mathrm{E}, 2086 \mathrm{~m}, \mathrm{v} .2011,(1 \mathrm{w})$, previous material is collected by

367 Sharaf, M. R. and deposited in KSMA; Shada Al A'la, $19.842917^{\circ}$ N, $41.311517^{\circ} \mathrm{E}, 1666$ m,

368 xii.2014, (2 w); Shada Al A'la, $19.842917^{\circ} \mathrm{N}, 41.311517^{\circ} \mathrm{E}, 1666 \mathrm{~m}$, vii.2015, (2 w); Shada Al

369 A'la, $19.845167^{\circ} \mathrm{N}, 41.30445^{\circ} \mathrm{E}, 1474 \mathrm{~m}$, iii.2015, (1 w); Shada Al A'la, $19.840183^{\circ} \mathrm{N}$,

$37041.311433^{\circ} \mathrm{E}, 1611 \mathrm{~m}$, vii.2015, (1 w); Shada Al A’la, $19.8511^{\circ} \mathrm{N}, 41.300617^{\circ} \mathrm{E}, 1325 \mathrm{~m}$,

371 iii.2015, (1 w); Shada Al A'la, $19.842917^{\circ} \mathrm{N}, 41.311517^{\circ} \mathrm{E}, 1666 \mathrm{~m}$, xi.2015, (1 w), previous 
372 material is collected using PT by Al Dhafer et al. Asir Province: Dalaghan park, $18.066066^{\circ} \mathrm{N}$, $37342.710981^{\circ} \mathrm{E}, 2223 \mathrm{~m}$, iv.2008, (2 w); Abha-Khamis Mushayt Road, $18.239186^{\circ} \mathrm{N}$,

$37442.588481^{\circ} \mathrm{E}, 2129 \mathrm{~m}$, ix.2004, (5 w); W. Bagara, $18.79214^{\circ} \mathrm{N}, 42.01912^{\circ} \mathrm{E}, 428 \mathrm{~m}$, vi.2014, (3 375 w); Abha-Khamis Mushayt Road, $18.23875^{\circ} \mathrm{N}, 42.570694^{\circ} \mathrm{E}, 2147 \mathrm{~m}$, iv.2011, (21 w); Abha376 Khamis Mushayt Road, $18.23875^{\circ} \mathrm{N}, 42.570694^{\circ} \mathrm{E}, 2147 \mathrm{~m}, \mathrm{x} .2014,(12 \mathrm{w})$; Raydah:

$37718.204267^{\circ} \mathrm{N}, 42.4124^{\circ} \mathrm{E}, 2820 \mathrm{~m}$, ii.2014, (12 w); $18.204267^{\circ} \mathrm{N}, 42.4124^{\circ} \mathrm{E}, 2820 \mathrm{~m}$, iv.2014, $378(4 \mathrm{w}) ; 18.22217^{\circ} \mathrm{N}, 42.40241^{\circ} \mathrm{E}, 2744 \mathrm{~m}$, vi.2014, PT (4 w), previous materials are collected by 379 Sharaf, M. R.; $18.204267^{\circ} \mathrm{N}, 42.4124^{\circ} \mathrm{E}, 2820 \mathrm{~m}$, vi.2014, $(1 \mathrm{w}) ; 18.204267^{\circ} \mathrm{N}, 42.4124^{\circ} \mathrm{E}, 2820$ $380 \mathrm{~m}$, viii.2014, (4 w); $18.204267^{\circ} \mathrm{N}, 42.4124^{\circ} \mathrm{E}, 2820 \mathrm{~m}$, iii. $2015,(2 \mathrm{w}) ; 18.20525^{\circ} \mathrm{N}$, $38142.410117^{\circ} \mathrm{E}, 2761 \mathrm{~m}$, ii.2014, (1 w); $18.20525^{\circ} \mathrm{N}, 42.410117^{\circ} \mathrm{E}, 2761 \mathrm{~m}$, iv.2014, (3 w); $38218.20525^{\circ} \mathrm{N}, 42.410117^{\circ} \mathrm{E}, 2761 \mathrm{~m}$, vi.2014, (3 w); $18.20525^{\circ} \mathrm{N}, 42.410117^{\circ} \mathrm{E}, 2761 \mathrm{~m}$, 383 viii.2014, ( $2 \mathrm{w}) ; 18.20525^{\circ} \mathrm{N}, 42.410117^{\circ} \mathrm{E}, 2761 \mathrm{~m}, \mathrm{i} .2015,(1 \mathrm{w}) ; 18.20525^{\circ} \mathrm{N}, 42.410117^{\circ} \mathrm{E}$, $3842761 \mathrm{~m}, \mathrm{i} .2015$, PT ( $2 \mathrm{w}) ; 18.20525^{\circ} \mathrm{N}, 42.410117^{\circ} \mathrm{E}, 2761 \mathrm{~m}$, vii.2015, (3 w); $18.20525^{\circ} \mathrm{N}$, $38542.410117^{\circ} \mathrm{E}, 2761 \mathrm{~m}$, xi.2015, (2 w); $18.198067^{\circ} \mathrm{N}, 42.40725^{\circ} \mathrm{E}, 2387 \mathrm{~m}$, ii.2014, (4 w); 386 $18.198067^{\circ} \mathrm{N}, 42.40725^{\circ} \mathrm{E}, 2387 \mathrm{~m}$, iv.2014, (1 w); $18.198067^{\circ} \mathrm{N}, 42.40725^{\circ} \mathrm{E}, 2387 \mathrm{~m}$, 26.viii.2014, (4 w); $18.198067^{\circ} \mathrm{N}, 42.40725^{\circ} \mathrm{E}, 2387 \mathrm{~m}, \mathrm{x} .2014,(3 \mathrm{w}) ; 18.198067^{\circ} \mathrm{N}$, 388

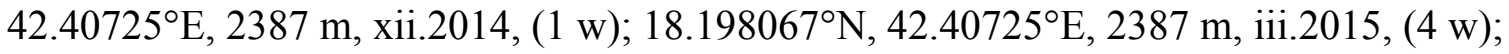
$18.198067^{\circ} \mathrm{N}, 42.40725^{\circ} \mathrm{E}, 2387 \mathrm{~m}$, iv.2015, (4 w); $18.198067^{\circ} \mathrm{N}, 42.40725^{\circ} \mathrm{E}, 2387 \mathrm{~m}$, vii.2015, (3 w); $18.201583^{\circ} \mathrm{N}, 42.408933^{\circ} \mathrm{E}, 2578 \mathrm{~m}$, ii.2014, (5 w); $18.201583^{\circ} \mathrm{N}, 42.408933^{\circ} \mathrm{E}$, $2578 \mathrm{~m}$, iv.2014, (3 w); $18.201583^{\circ} \mathrm{N}, 42.408933^{\circ} \mathrm{E}, 2578 \mathrm{~m}$, vi.2014, (7 w); $18.201583^{\circ} \mathrm{N}$, $42.408933^{\circ} \mathrm{E}, 2578 \mathrm{~m}$, viii.2014, (10 w); $18.201583^{\circ} \mathrm{N}, 42.408933^{\circ} \mathrm{E}, 2578 \mathrm{~m}, \mathrm{x} .2014$, (2 w); $18.201583^{\circ} \mathrm{N}, 42.408933^{\circ} \mathrm{E}, 2578 \mathrm{~m}$, iii.2015, $(1 \mathrm{w}) ; 18.201583^{\circ} \mathrm{N}, 42.408933^{\circ} \mathrm{E}, 2578 \mathrm{~m}$, v.2015, (5 w); $18.201583^{\circ} \mathrm{N}, 42.408933^{\circ} \mathrm{E}, 2578 \mathrm{~m}$, vii. $2015,(5 \mathrm{w}) ; 18.201583^{\circ} \mathrm{N}, 42.408933^{\circ} \mathrm{E}$, $2578 \mathrm{~m}$, xi. $2015,(2 \mathrm{w}) ; 18.1961^{\circ} \mathrm{N}, 42.40525^{\circ} \mathrm{E}, 2285 \mathrm{~m}$, ii. $2014,(2 \mathrm{w}) ; 18.1961^{\circ} \mathrm{N}$, 42.40525 ${ }^{\circ} \mathrm{E}, 2285 \mathrm{~m}$, viii.2014, (3 w); $18.1961^{\circ} \mathrm{N}, 42.40525^{\circ} \mathrm{E}, 2285 \mathrm{~m}, \mathrm{x} .2014,(2 \mathrm{w})$; $18.1961^{\circ} \mathrm{N}, 42.40525^{\circ} \mathrm{E}, 2285 \mathrm{~m}$, xi.2015, (2 w); $18.1961^{\circ} \mathrm{N}, 42.40525^{\circ} \mathrm{E}, 2285 \mathrm{~m}$, iii.2015, (5 w); $18.1961^{\circ} \mathrm{N}, 42.40525^{\circ} \mathrm{E}, 2285 \mathrm{~m}, \mathrm{i} .2015,(1 \mathrm{w}) ; 18.1961^{\circ} \mathrm{N}, 42.40525^{\circ} \mathrm{E}, 2285 \mathrm{~m}, \mathrm{v} .2015$, (3 w); $18.194917^{\circ} \mathrm{N}, 42.396967^{\circ} \mathrm{E}, 1897 \mathrm{~m}, \mathrm{x} .2014,(5 \mathrm{w}) ; 18.194917^{\circ} \mathrm{N}, 42.396967^{\circ} \mathrm{E}, 1897 \mathrm{~m}$, 400 iii.2015, (1 w); $18.194917^{\circ} \mathrm{N}, 42.396967^{\circ} \mathrm{E}, 1897 \mathrm{~m}$, vii. $2015,(8 \mathrm{w}) ; 18.194917^{\circ} \mathrm{N}$, $40142.396967^{\circ} \mathrm{E}, 1851 \mathrm{~m}$, xii. $2014,(1 \mathrm{w}) ; 18.195817^{\circ} \mathrm{N}, 42.389083^{\circ} \mathrm{E}, 1614 \mathrm{~m}$, ii.2014, (1 w); 402 $18.195817^{\circ} \mathrm{N}, 42.389083^{\circ} \mathrm{E}, 1614 \mathrm{~m}$, viii.2014, (5 w); $18.195817^{\circ} \mathrm{N}, 42.389083^{\circ} \mathrm{E}, 1614 \mathrm{~m}$, 
403 xii.2014, (5 w); $18.195817^{\circ} \mathrm{N}, 42.389083^{\circ} \mathrm{E}, 1614 \mathrm{~m}, \mathrm{i} .2015,(2 \mathrm{w}) ; 18.195817^{\circ} \mathrm{N}, 42.389083^{\circ} \mathrm{E}$, $4041614 \mathrm{~m}$, iii.2015, (3 w); $18.195817^{\circ} \mathrm{N}, 42.389083^{\circ} \mathrm{E}, 1614 \mathrm{~m}$, vii.2015, (1 w); $18.195817^{\circ} \mathrm{N}$, $40542.389083^{\circ} \mathrm{E}, 1614 \mathrm{~m}, \mathrm{ix} .2015$, (3 w); $18.195817^{\circ} \mathrm{N}, 42.389083^{\circ} \mathrm{E}, 1614 \mathrm{~m}$, xi.2015, (3 w); $40618.193633^{\circ} \mathrm{N}, 42.390333^{\circ} \mathrm{E}, 1772 \mathrm{~m}, \mathrm{iv} .2014,(1 \mathrm{w}) ; 18.193633^{\circ} \mathrm{N}, 42.390333^{\circ} \mathrm{E}, 1772 \mathrm{~m}$, 407 viii.2014, (1 w); $18.193633^{\circ} \mathrm{N}, 42.390333^{\circ} \mathrm{E}, 1772 \mathrm{~m}, \mathrm{x} .2014,(2 \mathrm{w}) ; 18.193633^{\circ} \mathrm{N}$, $40842.390333^{\circ} \mathrm{E}, 1772 \mathrm{~m}$, xii.2014, (1 w); $18.193633^{\circ} \mathrm{N}, 42.390333^{\circ} \mathrm{E}, 1772 \mathrm{~m}, \mathrm{i} .2015$, (1 w); $40918.193633^{\circ} \mathrm{N}, 42.390333^{\circ} \mathrm{E}, 1772 \mathrm{~m}$, iii.2015, $(1 \mathrm{w}) ; 18.193633^{\circ} \mathrm{N}, 42.390333^{\circ} \mathrm{E}, 1772 \mathrm{~m}$, 410 v.2015, (1 w); $18.193633^{\circ} \mathrm{N}, 42.390333^{\circ} \mathrm{E}, 1772 \mathrm{~m}, \mathrm{vi} .2015,(3 \mathrm{w}) ; 18.193633^{\circ} \mathrm{N}, 42.390333^{\circ} \mathrm{E}$, $4111772 \mathrm{~m}$, vii.2015, (1 w); $18.193633^{\circ} \mathrm{N}, 42.390333^{\circ} \mathrm{E}, 1772 \mathrm{~m}$, ix.2015, (2 w), previous material 412 is collected using PT by Al Dhafer et al. and deposited in KSMA; Riyadh Province: Riyadh, 413 Takhassosy street, $24.693472^{\circ} \mathrm{N}, 46.671136^{\circ} \mathrm{E}, 615 \mathrm{~m}, \mathrm{x} .2015,(1 \mathrm{w})$; Riyadh, Alhayer, $41424.280194^{\circ} \mathrm{N}, 46.765861^{\circ} \mathrm{E}$, iii.2009, (6 w); Dirab, KSU research farm, $24.4085^{\circ} \mathrm{N}$, $41546.661639^{\circ} \mathrm{E}, 588 \mathrm{~m}$, xii.2009, (1 w); Oyaina, $24.90665^{\circ} \mathrm{N}, 46.389917^{\circ} \mathrm{E}, 749 \mathrm{~m}$, iv.2010, (3 w); 416 Qarina, $25.132275^{\circ} \mathrm{N}, 46.163883^{\circ} \mathrm{E}, 761 \mathrm{~m},(3 \mathrm{w})$; Riyadh, Al Emam University campus, $41724.809158^{\circ} \mathrm{N}, 46.701892^{\circ} \mathrm{E}, 650 \mathrm{~m}, \mathrm{x.2010}$, (1 w); Riyadh, KSU guest building, Dereyia, $41824.716667^{\circ} \mathrm{N}, 46.616667^{\circ} \mathrm{E}, 612 \mathrm{~m}$, vii.2009, (1 w), previous material is collected by Sharaf, M. 419 R. and deposited in KSMA; Riyadh, Janaderiyah, $24.98121^{\circ} \mathrm{N}, 46.77711^{\circ} \mathrm{E}, 630 \mathrm{~m}, \mathrm{ix} .2014,(1$ 420 w); Riyadh, Hawtet Sudair, 25.60490 N, 45.60050E, 751 m, i.2015, (1 w); Hawtet Bani Tamim, $42123.48019^{\circ} \mathrm{N}, 46.84350^{\circ} \mathrm{E}, 597 \mathrm{~m}, \mathrm{i} .2014$, (3 w); Hawtet Bani Tamim, 23.50737 ${ }^{\circ} \mathrm{N}, 46.90059^{\circ} \mathrm{E}$, $422593 \mathrm{~m}$, xii.2014, (10 w); Hawtet Bani Tamim, 23.45943ํN, 46.81895 E, $582 \mathrm{~m}$, xii.2014, (4 w); 423 Riyadh, Ammariya, 24.81839º N, 46.44698 ${ }^{\circ} \mathrm{E}, 696 \mathrm{~m}, \mathrm{x} .2013,(11 \mathrm{w})$; Mezahmiya, 24.45570 $\mathrm{N}$, $42446.14881^{\circ} \mathrm{E}, 719 \mathrm{~m}, \mathrm{x} .2013,(42 \mathrm{w})$; Mezahmiya, 24.47197º N, 46.23878 ${ }^{\circ} \mathrm{E}, 633 \mathrm{~m}, \mathrm{i} .2014,(2$ 425 w); Dirab, KSU research farm, $24.4085^{\circ} \mathrm{N}, 46.661639^{\circ} \mathrm{E}, 588 \mathrm{~m}$, xii.2013, (4 w); Layla (Alaflaj), $42622.21325^{\circ} \mathrm{N}, 46.68818^{\circ} \mathrm{E}, 543 \mathrm{~m}, \mathrm{i} .2014,(4 \mathrm{w})$; Elsolayel, $20.45473^{\circ} \mathrm{N}, 45.57120^{\circ} \mathrm{E}, 616 \mathrm{~m}$, 427 i.2014, (2 w); Riyadh, W. Hanifa, $24.67089^{\circ} \mathrm{N}, 46.58061^{\circ} \mathrm{E}, 654 \mathrm{~m}$, ii.2014, (3 w); Riyadh, W. 428 Hanifa, $24.77091^{\circ} \mathrm{N}, 46.53147^{\circ} \mathrm{E}, 695 \mathrm{~m}$, ix.2014, (10 w); Riyadh, W. Hanifa, $24.74747^{\circ} \mathrm{N}$, $42946.56474^{\circ} \mathrm{E}, 679 \mathrm{~m}$, ix.2014, (4 w); Riyadh, W. Hanifa, $24.73507^{\circ} \mathrm{N}, 46.57518^{\circ} \mathrm{E}, 674 \mathrm{~m}$, 430 ix.2014, (2 w); Huraymila, 25.12636 N, 46.15782 ${ }^{\circ} \mathrm{E}, 951 \mathrm{~m}$, ii.2014, (2 w); Dhurma, $43124.59961^{\circ} \mathrm{N}, 46.15547^{\circ} \mathrm{E}, 657 \mathrm{~m}$, iv.2014, (2 w); Thadiq, $25.30974^{\circ} \mathrm{N}, 45.86457^{\circ} \mathrm{E}, 736 \mathrm{~m}$, 432 iv.2014, (2 w); Thadiq, 25.29360 $\mathrm{N}, 45.87102^{\circ} \mathrm{E}, 735 \mathrm{~m}, \mathrm{iv} .2014$, (2 w); Quwaiiyah, $43324.04718^{\circ} \mathrm{N}, 45.24430^{\circ} \mathrm{E}, 854 \mathrm{~m}, \mathrm{v} .2014$, (3 w); Quwaiiyah, $24.04347^{\circ} \mathrm{N}, 45.24007^{\circ} \mathrm{E}, 857 \mathrm{~m}$, 
434 v.2014, (3 w); Shaqra, 25.26655 ${ }^{\circ} \mathrm{N}, 45.26779^{\circ} \mathrm{E}, 728 \mathrm{~m}, \mathrm{v} .2014$, (2 w); Shaqra, $25.32638^{\circ} \mathrm{N}$, $43545.23341^{\circ} \mathrm{E}, 710 \mathrm{~m}, \mathrm{v} .2014,(2 \mathrm{w})$; Shaqra, $25.23018^{\circ} \mathrm{N}, 45.31915^{\circ} \mathrm{E}, 703 \mathrm{~m}, \mathrm{i} .2015,(3 \mathrm{w})$; $\mathrm{Al}$

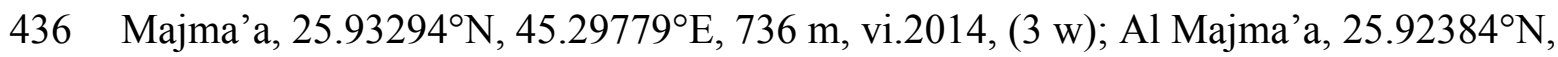
$43745.38035^{\circ} \mathrm{E}, 743 \mathrm{~m}$, ii.2014, (5 w); Al Ghat, 26.02495 ${ }^{\circ} \mathrm{N}, 44.93650^{\circ} \mathrm{E}, 670 \mathrm{~m}$, vi.2014, (3 w); $\mathrm{Al}$ 438 Ghat, $26.06582^{\circ} \mathrm{N}, 44.91929^{\circ} \mathrm{E}, 653 \mathrm{~m}$, x.2015, (2 w); Riyadh-Al Kharj Road, $24.29615^{\circ} \mathrm{N}$, $43947.15553^{\circ} \mathrm{E}, 453 \mathrm{~m}$, ix.2014, (4 w); W. Al Dawasir, 20.49055 N, 44.79462 ${ }^{\circ} \mathrm{E}, 721 \mathrm{~m}$, ii.2015, (2 440 w), previous material is collected by Salman, S. and deposited in KSMA; Jazan Province: Abu 441 Arish, $17.01347^{\circ} \mathrm{N}, 42.80160^{\circ} \mathrm{E}, 90 \mathrm{~m}$, iv.2012, (6 w); W. Shahdan, $17.45222^{\circ} \mathrm{N}, 42.71516^{\circ} \mathrm{E}$,

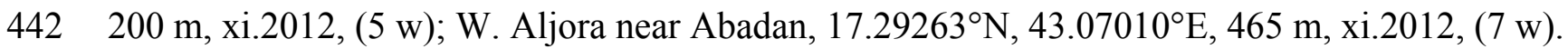
443 Eastern Province: Al Qatif, El Naft, $26.510278^{\circ} \mathrm{N}, 49.968889^{\circ} \mathrm{E}, 30 \mathrm{~m}$, iii.2012, (2 w) All 444 previous material is collected by Sharaf M. R. and deposited in KSMA; Wadi Shugub, 1390 m, 445 iv.1983, (1 w); Al Tawlah, 1.iv.1983, (1 w); Annamas, 8.iv.1983, (1 w); Hufuf, 14.iv.1983, (1 446 w), previous material is collected by C. A. Collingwood and deposited in WMLC; TANZANIA: 447 Kizimgani, 16.iii.1988, (M. J. Ways), (3 w); Sotale, x.1989, (Lohr), (2 w); Kanga, x.1989, 448 (Lohr), (1 w).UNITED ARAB EMIRATES: Khor al-khwair, $25.95^{\circ} \mathrm{N}, 56.05^{\circ} \mathrm{E}, 08 . i i i-$ 449 07.v.2007, (M. Hauser et al.), UAE13173, CASENT0264114 (1 w, KSMA); Sharjah, 25.35N, $450 \quad 55.4^{\circ} \mathrm{E}, 28.1 \mathrm{i}-12.1 \mathrm{v} .2011$, (M. Hauser et al. ), UAE13028, CASENT0264785 (1 w, KSMA); W. 451 Bih dam, 25.8 N, 56.066667º E, 16-31.xii.2009, (M. Hauser et al. ), UAE13123, 452 CASENT0264962 (1 w, KSMA); Dubai, palm Deira trees by metro, $25.276^{\circ} \mathrm{N}, 55.300^{\circ} \mathrm{E}$, 453 16.v.2012, (Wetterer J.), (2 w, \#99, \#107, KSMA); no locality, iii.2005, (Collingwood ), 454 UAE173, (1 w, WMLC); ZIMBABWE: Bulawayo, 04.xii.1916, (G. Arnold) (18 Syntype w); 455 Sawmills, 22.xi.1918, (G. Arnold), (3 w, 2 m); Victoria Falls, 09.xii.1914, (G. Arnold), (7 w). 456 Description

457 Head. In full-face view distinctly longer than broad with nearly parallel sides and feebly concave 458 of straight posterior margin; clypeal carinae feebly developed, broadly separated and clearly 459 divergent anteriorly; anterior clypeal margin feebly concave, without anterior sharp angles; 460 antennae 11-segmented; scapes, when laid back straight from their insertions, failing to reach 461 posterior margin of head; eye size variable (EL 0 19-0.22 $\times \mathrm{HW}$ ), in profile consisting of an 462 outer ring of ommatidia encloses one or two longitudinal rows of 2-4 ommatidia; in few 463 specimens one or two ommatidia within the ring present; eyes distinctly situated in front of 464 midlength of head sides in full-face view. Mesosoma. Promesonotum feebly convex in profile; 
465 metanotal groove distinctly impressed, with short cross-ribs; propodeal dorsum and declivity 466 meeting in a rounded convexity. Petiole. Petiolar peduncle short, with a small anteroventral 467 process in profile; petiolar node low-subconical in profile. Postpetiole. Postpetiole smaller than 468 petiole, lower and broadly convex dorsally in profile. Sculpture. Entire body smooth and 469 shining, except for metanotal cross-ribs on sides of metanotal groove. Pilosity. All body surfaces 470 with standing hairs, pronotum with a single pair on anterior margin between humeral pair;

471 promesonotum usually with four pairs of hairs but in many specimens a fifth pair present;

472 propodeum without hairs or with one or two pairs of hairs. Color. Variable, from uniform yellow 473 to uniform dark brown, frequently with a pair of brown patches or a darker band apically on first 474 gastral tergite.

475 Note

476 Aldawood and Sharaf (2011) described M. dryhimi from Al Baha Province (KSA)

477 based on the worker caste. Comparing the type material of M. dryhimi and M. montanum with M. 478 exiguum revealed that they have similar body size and color, in addition to the possession of the 479 following characters: eyes of moderate size, with an outer ring of ommatidia encloses one or two 480 longitudinal rows of 2-4 ommatidia; metanotal groove impressed, with distinct cross-ribs; all 481 body surfaces with standing hairs, pronotum with a single pair on anterior margin between 482 humeral pair; posterior half of first gastral tergite brown; body smooth and shining. Herein, we 483 treat M. dryhimi and M. montanum as junior synonyms of M. exiguum.

\section{Biological and Ecological notes}

485 This species is by far the most common Arabian species of the M. monomorium-group, and it 486 appears to be very flexible in its ecological requirements since it occurs in numerous habitats 487 throughout the Arabian Peninsula. It was found living in humid soil, leaf litter, under rocks, and 488 under bark. Once it was even collected from inside galleries of a Camponotus sp. colony.

489 Furthermore, M. exiguum was found in a variety of agricultural landscapes and human 490 settlements, very often in close proximity to trees or other vegetation.

\section{Geographic range}

492 Even though this species was originally described from Ethiopia (Forel, 1894), M. exiguum is 493 extremely widespread (Table 1) since it occurs in much of the Afrotropical Region (Bolton, 494 1987; Sharaf et al., 2017), the Malagasy Region (Heterick, 2006), and the Mediterranean Basin 495 (Gòmez \& Espadaler, 2006, Sharaf, 2006). As mentioned above, this species is also broadly 
496 distributed throughout the Arabian Peninsula, and is considered as the commonest species of the

497 M. monomorium-group in the region. The first record from the Arabian Peninsula was from the

498 KSA (Aldawood \& Sharaf, 2009) while later records from the UAE, Oman, and Yemen

499 (Collingwood et al. 2011) were published under the name M. baushare Collingwood \& Agosti,

5001996 that was recently synonymized under M. exiguum (Sharaf et al., 2017).

501 Monomorium holothir Bolton, 1987, Figure 8

502 Monomorium holothir Bolton, 1987: 393 (w.)

503 Type material examined

504 Holotype, pinned worker, KENYA: Lake Baringo, 1.XII 1983 (L. Darlington) (BMNH:

505 CASENT0902243). Paratype, pinned worker with same data as holotype (BMNH).

506 Non-type material examined

507 KSA: Jazan, $16.97627^{\circ} \mathrm{N}, 42.61743^{\circ} \mathrm{E}$, alt. $38 \mathrm{~m}, 12 . \mathrm{iv} .2012$, (Sharaf M. R.) (4 w, KSMA)

508 (CASENT0906392); Jazan, Abu Arish, 17.01347N, 42.80160 E, alt. 90 m, 10.iv.2012, (Sharaf

509 M. R.) (1 w, KSMA); Jazan, Zabia, $17.10745^{\circ} \mathrm{N}, 42.65026^{\circ} \mathrm{E}$, alt. 43 m, 9.iv.2012, (Sharaf M.

510 R.) (4 w, KSMA).

\section{Description}

512 Head. In full-face view distinctly longer than broad with feebly convex sides behind eyes and

513 nearly straight or feebly concave posterior margin; clypeal carinae sharply developed; anterior

514 clypeal margin feebly concave; antennae 12-segmented; scapes failing to reach posterior margin

515 of head; eyes relatively large (EL $0 \cdot 30 \times \mathrm{HW}$ ), with 7-9 ommatidia in longest row; in profile eye

516 length clearly greater than distance between anteriormost point of eyes and nearest point of

517 mandibular insertion. Mesosoma. Promesonotum feebly convex in profile; metanotal groove

518 distinctly impressed, with distinct cross-ribs; propodeal dorsum and declivity meeting in a

519 rounded convexity; propodeal spiracle small and pinhole-like. Petiole. Petiolar node high,

520 subconical and with narrowly rounded dorsum in profile. Sculpture. Entire body smooth and

521 shining, except for metanotal cross-ribs on sides of metanotal groove. Pilosity. All body surfaces

522 with abundant long-standing hairs; promesonotum with more than six pairs of hairs; propodeum

523 with four pairs of hairs. Color. Variable, from yellow to brown-yellow.

\section{Biological and Ecological notes}

525 This species was collected from mangos, Mangifera sp. (Anacardiaceae) imported from Kenya

526 and this provides the context for the present record. It was also found in leaf litter next to 
527 Calotropis procera (Aiton) W.T.Aiton (Asclepiadaceae). Some workers were found nesting in a

528 thin layer of clay soil above sandy soil, while several workers were collected from leaf litter

529 under a Conocarpus L. tree (Combretaceae).

530 Geographic range

531 Monomorium holothir is a comparatively rare species originally described from Kenya and prior

532 to this study only known from the type locality (Bolton, 1987; Hita Garcia et al, 2013). Our

533 collections represent a new species record for KSA, and it is highly likely an introduction to the

534 country.

535 Monomorium mohammedi Sharaf \& Hita Garcia sp. n., Figure 9

536 Type material

537 Holotype, pinned worker, KSA: Almajardah, Wadi Khat, $19.08913^{\circ} \mathrm{N}, 41.97126^{\circ} \mathrm{E}$, alt. $513 \mathrm{~m}$,

538 10.xi.2012, (Sharaf M. R.) (KSMA: CASENT0823774). Paratypes, six pinned workers: KSA: 2

539 w, with same data as the holotype (KSMA); 3 w, Jazan, Wadi Shahdan, $17.45222^{\circ} \mathrm{N}$,

$54042.71516^{\circ} \mathrm{E}$, alt. $200 \mathrm{~m}, 13 . x i .2012$, (Sharaf M. R.) (1 in CASC: CASENT0922351, 2 in

541 KSMA); 1 w, Jazan, Abu Arish, $17.01347^{\circ}$ N, $42.80160^{\circ}$ E, alt. 90 m, 10.iv.2012, (Sharaf M. R.)

542 (WMLC).

543 Measurements

544 Holotype: TL 1.37; HL 0.41; HW 0.31; SL 0.25; EL 0.04; EM 0.09; ML 0.42; PW 0.19; PTL

545 0.15; PTW 0.08; PTH 0.11; PPL 0.07; PPW 0.08; PPH 0.08; CI 76; EI 13; SI 81.

546 Paratypes ( $\mathrm{n}=7$ ): TL 1.32-1.53; HL 0.38-0.44; HW 0.29-0.32; SL 0.26-0.29; EL 0.04-0.05;

547 EM 0.09-0.11; ML 0.36-0.44; PW 0.18-0.21; PTL 0.08-0.11; PTW 0.07-0.08; PTH 0.09-0.12;

548 PPL 0.05-0.07; PPW 0.08; PPH 0.08; CI 71-82; EI 13-17; SI 88-97.

549 Diagnosis. Monomorium mohammedi can be readily diagnosed by the combination of the

550 following characters: eyes distinctly small, with 5-6 ommatidia; mesosoma, petiole, and

551 postpetiole without standing hairs; mesopleuron, metapleuron, petiole and postpetiole finely

552 shagreened.

553 Description

554 Worker. Head. In full-face view distinctly longer than broad with shallowly convex or nearly

555 parallel sides and clearly concave posterior margin in full-face view; median clypeal portion

556 without carina or anterolateral angles, anterior clypeal margin feebly concave; antenna 11-

557 segmented; scapes short, when laid straight back, just surpassing midlength of head (SI 88-97); 
558 mandibles armed with three teeth, decreasing in size from apex to base; eyes oval, tiny, (EL

$5590.13-0.15 \times \mathrm{HW}$ ) with 5 ommatidia, set in front of midlength of head; frontal lobes farther apart

560 in full-face view; underside of head with six scattered short hairs. Mesosoma. In profile with a

561 flat promesonotal dorsum, which slopes posteriorly to a well-defined metanotal groove;

562 propodeal spiracles small and pinhole-like; propodeal dorsum evenly sloping posteriorly to short

563 declivity. Petiole: Node massive, narrowly rounded above, and little higher than postpetiolar

564 node in profile; anterior peduncle short; ventral petiolar surface below node broadly convex

565 extending anteriorly to form a blunt broad dent. Postpetiole. Node small with convex dorsal

566 margin; postpetiole as high as broad. Sculpture. Cephalic surface smooth and shining;

567 mandibles smooth and shining, with faint striations on the outer margin; mesosoma dorsum and

568 propleuron smooth and shining; meso-and metapleuron finely shagreened; metanotal cross ribs

569 distinct; petiole and postpetiole with traces of superficially shagreened sculpture, but never

570 smooth; gaster smooth and shining. Pilosity. Underside of head without hairs; cephalic surface

571 with scattered minute hair-pits; anterior clypeal margin and mandibles with longer hairs;

572 antennae with abundant appressed hairs; mesosoma without hairs, only rare appressed

573 pubescence; petiole and postpetiole without hairs, only few appressed pubescence dorsally;

574 gaster with scattered appressed pubescence, few longer hairs on the last gastral tergites. Color.

575 Overall uniform clear yellow, mandibular teeth light brown.

576 Differential diagnosis

577 This new species is closest to M. guillarmodi Arnold, 1946 from Lesotho in terms of the small

578 body size, tiny eyes, 11-segmented antennae, lack of hairs on mesosoma, and the smooth body.

579 However, M. mohammedi is readily separated from M. guillarmodi by the following characters:

580 the posterior margin of head without hairs and concave in full-face view, petiole and postpetiole

581 without hairs, median clypeal portion without anterolateral angles or carina, scape relatively

582 longer (SI 81-97), whereas M. guillarmodi has a transverse posterior head margin with 1-2 pairs

583 of hairs, petiole and postpetiole each with a single pair of hairs, median clypeal portion

584 prominent with well-defined anterolateral angles and distinct carina, and scape shorter (SI less

585 than 80 ).

586 Among the Arabian species of the M. monomorium-group only three species have 11-segmented

587 antennae: M. mohammedi, M. clavicorne, M. aeyade, and M. exiguum. Monomorium

588 mohammedi is easily separated from clavicorne by its smaller eyes, smaller terminal funicular 
589 segment, and lack of mesosomal pilosity, whereas clavicorne has larger eyes, greatly swollen

590 terminal funicular segments, and abundant hairs on the mesosoma.

591 When comparing M. mohammedi with M. aeyade, both lack hairs on the mesosoma but $M$.

592 mohammedi can be immediately separated by its smaller eyes with only 5 ommatidia that are

593 situated distinctly further apart from the mandibular insertions (EM 0.09-0.11), the finely

594 shagreened meso- and metapleuron, the hairless petiole and postpetiole, whereas M. aeyade has

595 larger eyes (EL $0.24 \times \mathrm{HW}$ ), with a ring of ommatidia encircling a single row of 2 ommatidia

596 that are situated closer to the mandibular insertions (EM 0.05); meso-and metapleuron smooth,

597 and petiole and postpetiole each with one pair of hairs.

598 Biological and Ecological notes

599 The new species was collected from leaf litter under a Hyphaene tree (Arecaceae) and another

600 nest series was found in a thin layer of clay soil above sandy soil under a Mango tree.

601 Geographic range

602 So far, the new species is only known from the type locality.

603 Etymology

604 The name of the new species is a patronym in honor of Mohammed Sharaf, the eight year old

605 son of the senior author.

606 Monomorium sarawatense Sharaf \& Aldawood, 2013, Figure 10

607 Monomorium sarawatensis Sharaf \& Aldawood, 2013: 70, figs. 5-15 (w).

608 Type material examined

609 Holotype, pinned worker, KSA: Al-Baha Province, Aqabet Al-Baha-Tihama, 20.00000N,

$61041.43758^{\circ} \mathrm{E}, 1300 \mathrm{~m}$ ), 19.iv.2012 (Sharaf M. R.) (KSMA). Paratypes, 26 pinned workers with

611 same data as holotype (one in BMNH, 25 in KSMA).

612 Non-type material examined

613 KSA: Al Baha Province, Aqabet Al-Baha-Tihama, 20.00000 N, 41.43758 ${ }^{\circ} \mathrm{E}, 1300 \mathrm{~m}$,

614 19.iv.2012 (Sharaf M. R.) (21 w, KSMA); Shada Al A’la, $19.8511^{\circ} \mathrm{N}, 41.300617^{\circ} \mathrm{E}, 1325 \mathrm{~m}$,

615 2.iii.2015, (Al Dhafer et al.) (1 w, KSMA); Asir Province: Raydah, $18.1961^{\circ} \mathrm{N}, 42.40525^{\circ} \mathrm{E}$,

6162285 m, 26.iii.2014, Malaise trap (Al Dhafer et al.) (1 w, KSMA).

617 Description

618 Head. Distinctly longer than broad, with nearly straight posterior margin and shallowly convex

619 sides; anterior clypeal margin feebly concave between a pair of obtusely projecting angles; 
620 clypeal carinae broadly separated and subparallel; antenna 12-segmented; scapes, when laid back

621 from their insertions, fail to reach posterior margin of head; eyes with five-six ommatidia in

622 longest row (EL $0.17-0.22 \times \mathrm{HW}$ ); with head in profile the posterior margins of eyes at

623 midlength of sides. Mesosoma. In profile with promesonotum straight or feebly convex;

624 metanotal groove deep and broad; propodeal dorsum making weak obtuse angle with propodeal

625 declivity; propodeal spiracle small and pinhole-like. Petiole. Petiolar node high and acuminate in

626 profile, petiolar peduncle thick and short. Postpetiole. In dorsal view clearly broader than long.

627 Sculpture. Cephalic dorsum smooth and shining; genae faintly longitudinally striate; mesosoma

628 densely reticulate-punctate except for pronotal sides, which are nearly smooth and shining;

629 petiole and postpetiole densely reticulate-punctate. Pilosity. Body pilosity clubbed; cephalic

630 dorsum with few scattered hair-pits; mesosomal pilosity few and sparse, two pairs of erect setae

631 on pronotum, five or more on mesonotum, three on propodeum, petiole usually with two pairs of

632 erect setae. Color. Uniformly yellow.

633 Biological and Ecological notes

634 Monomorium sarawatense was found nesting inside woody fruits of Annona squamosal L.

635 (Annonaceae).

\section{Geographic range}

637 Based on current knowledge, this species is endemic to the southwestern Mountains of the KSA.

638 DISCUSSION

639

640 The previously documented number of species of the M. monomorium-group from the Arabian

641 Peninsula was six: M. aeyade,_M. clavicorne, M. dryhimi, M. exiguum, M. montanum, and

642 M. sarawatense (Collingwood, 1985; Collingwood \& Agosti, 1996; Aldawood \& Sharaf, 2009;

643 Aldawood \& Sharaf, 2011; El-Hawagry et al., 2013; Sharaf et al., 2015 \& Sharaf et al., 2017). In

644 this study, the number of species remains six due to some taxonomic amendments, additional

645 records, and the descriptions of M. mohammedi sp. n.

646 Within the M. monomorium-group, M. exiguum is one of the most broadly distributed species

647 (Figure 11A) due to its ability to inhabit a broad range of habitats in the region. The species is

648 widespread in the central and southwestern regions of the KSA, Oman and the UAE but it is

649 highly likely that it has a similar broad geographical distribution in other unexplored areas of the

650 Arabian Peninsula. In addition, the species has a wide distribution range in the Afrotropical 
651 region (Table 1). The recent synonymies of some Arabian species under M. exiguum (Sharaf et 652 al., 2017), as well as the ones suggested in this study, reveal a remarkable size and color 653 variation, which has led to erroneous species descriptions in the past. During the present work, it 654 was observed that some nest series are uniform yellow, or with brown transverse bands on the 655 first and second gastral tergites, whereas other series have brown heads and yellow-brown 656 gasteral tergites. These variations were already noted by Bolton (1987) for the Afrotropical 657 fauna. These morphological variations should be taken into consideration in future studies that 658 will treat the $M$. monomorium-group in the region, especially those concerning descriptions of 659 new species, in order to avoid possible synonymies. Considering the challenging morphological 660 species delimitations, integrative approaches combining morphological and molecular techniques 661 might provide a more comprehensive taxonomic system (Schlick-Steiner et al., 2010).

662 The distribution of the three species M. holothir, M. mohammedi, and M. sarawatense (Figure 11 663 B) appears to be confined to the southwestern region of the KSA. The latter region is known for 664 its Afrotropical affinities (Bolton, 1994; Eig, 1938; Zohary, 1973; Aldawood et al., 2011; Sharaf 665 \& Aldawood, 2012; Sharaf et al., 2012a, b; El-Hawagryi et al., 2013, Sharaf \& Aldawood, 2013), 666 and the newly recorded Monomorium holothir, a species_previously only known from Kenya, 667 emphasizes this Afrotropical influence.

668

669 We hope that this work will help regional researchers with the identification of this rather 670 challenging group of ants and we also expect adding more material including new records and 671 undescribed species with further surveys of poorly collected areas of the region.

672 Conclusions. The Arabian ant fauna of the Monomorium monomorium species-group is revised,

673 keyed and illustrated based on the worker caste. Six species are treated with description of a new 674 species M. mohammedi sp. n. from the southwestern region of the KSA.

\section{ACKNOWLEDGEMENTS}

676 We thank Brian Heterick and an anonymous reviewer for useful comments. We are grateful to 677 Brian Fisher and Michele Esposito for imaging species. Mostafa Sharaf thanks Stephen Judd and 678 Tony Hunter for the help during the visit to the World Museum Liverpool, U. K. Special thanks 679 to Boris Kondratieff for careful editing of earlier version of the manuscript. Thanks also to 680 Andrew Polaszek for proving some material from Oman. 
682 Aldawood AS, Sharaf MR. 2009. Two ant species (Hymenoptera: Formicidae) as new records to

683

684

685

686

687

688

689

690

691

692

693

694

695

696

697

698

699

700

701

702

703

704

705

706

707

708

709

710

711 fauna of Saudi Arabia. Bulletin of Entomological Society of Egypt 86: 143-147.

Aldawood SA, Sharaf MR. 2011. Monomorium dryhimi sp. n., a new ant species (Hymenoptera, Formicidae) of the M. monomorium group from Saudi Arabia, with a key to the Arabian Monomorium monomorium-group. ZooKeys 106:47-54.

Aldawood AS, Sharaf MR, Taylor B. 2011. First record of the myrmicine ant genus Carebara Westwood, 1840 (Hymenoptera, Formicidae) from Saudi Arabia with description of a new species C. abuhurayri sp. n. ZooKeys 92: 61-69. http://dx.doi.org/10.3897/zookeys.92.770

André E. 1881. Catalogue raisonné des Formicides provenant du voyage en Orient de M. Abeille de Perrin et description des espèces nouvelles. Annales de la Société Entomologique de France (6)1:53-78.

Bolton B. 1987. A review of the Solenopsis genus-group and revision of Afrotropical Monomorium Mayr (Hymenoptera: Formicidae). Bulletin of the British Museum (Natural History). Entomology 54:263-452. Bolton B. 1994. Identification Guide to the Ant Genera of the World: Cambridge, Mass, $222 \mathrm{pp}$.

Bolton B. 2017. An online catalog of the ants of the world. Available at http://antcat.org/ [accessed 25 May 2017].

Brandão CRF. 2000. Specimen processing, building and curating an ant 797 collection Major regional and type collections of ants (Formicidae) of the world and 798 sources for the identification of ant species. In: Agosti D. et al. (eds) Ants. Standard 799 Methods for Measuring and Monitoring Biodiversity. Biological Diversity Handbook 800 Series 3: 17255-18571. Smithsonian Institution Press, Washington and London.

Brown WL Jr. 2000. Diversity of ants. Pp 45-79. In: Agosti et al. (eds.) Ants. Standard methods for measuring and monitoring biodiversity, Biological diversity hand book series. Smithsonian Institution Press, Washington D. C. 280 pp.

Collingwood CA. 1985. Hymenoptera: Fam. Formicidae of Saudi Arabia. Fauna of Saudi Arabia 7: $230-302$.

Collingwood CA, Agosti D. 1996. Formicidae (Insecta: Hymenoptera) of Saudi Arabia (part 2). Fauna of Saudi Arabia 15:300-385. 
712 Collingwood CA, Agosti D, Sharaf MR, Harten A. 2011. Order Hymenoptera, Family

713

714

715

716

717

718

719

720

721

722

723

724

725

726

727

728

729

730

731

732

733

734

735

736

737

738

739

740

741

Formicidae. Arthropod Fauna of the United Arab Emirates 4: 405-474.

Eig A. 1938. Taxonomic studies on the Oriental species of the genus Anthemis. Palestine Journal of Botany, Jerusalem 1: 161-224.

El-Hawagryi MS, Khalil MW, Sharaf MR, Fadl HH, Aldawood AS. 2013. A preliminary study on the insect fauna of Al-Baha Province, Saudi Arabia, with descriptions of two new species. Zookeys 274:1-88.

Emery C. 1908. Beiträge zur Monographie der Formiciden des paläarktischen Faunengebietes. (Hym.) Teil V. Deutsche Entomologische Zeitschrift 1908:663-686.

Emery C. 1922. Hymenoptera. Fam. Formicidae. Subfam. Myrmicinae. [part]. Genera Insectorum 174B:95-206.

Evenhuis, N. L. 2009. The Insect and Spider Collections of the World Website. http://hbs.bishopmuseum.org/codens/. [accessed 3 December 2017].

Forel A. 1894. Abessinische und andere afrikanische Ameisen, gesammelt von Herrn Ingenieur Alfred Ilg, von Herrn Dr. Liengme, von Herrn Pfarrer Missionar P. Berthoud, Herrn Dr. Arth. Müller etc. Mitteilungen der Schweizerischen Entomologischen Gesellschaft 9:64100.

Forel A. 1913a. Quelques fourmis du Musée du Congo Belge (1). Annales de la Société Entomologique de Belgique 57:347-359.

Forel A. 1913b. Ameisen aus Rhodesia, Kapland usw. (Hym.) gesammelt von Herrn G. Arnold, Dr. H. Brauns und Anderen. Deutsche Entomologische Zeitschrift 1913(Suppl.):203-225.

Forel A. 1916. Fourmis du Congo et d'autres provenances récoltées par MM. Hermann Kohl, Luja, Mayné, etc. Revue Suisse de Zoologie 24:397-460.

Gòmez K, Espadaler X. 2006. Exotic ants (Hymenoptera: Formicidae) in the Balearic Islands. Myrmecologische Nachrichten 8:225-233.

Heterick BE. 2001. Revision of the Australian ants of the genus Monomorium (Hymenoptera: Formicidae). Invertebrate Taxonomy 15:353-459.

Heterick B. 2006. A revision of the Malagasy ants belonging to genus Monomorium Mayr, 1855 (Hymenoptera: Formicidae). Proceedings of the California Academy of Sciences (4) 57:69-202. 
742 Hita Garcia F, Wiesel E, Fischer G. 2013. The Ants of Kenya (Hymenoptera: Formicidae) -

743

744

745

746

747

748

749

750

751

752

753

754

755

756

757

758

759

760

761

762

763

764

765

766

767

768

769

770

771

Faunal overview, first species checklist, bibliography, accounts for all genera, and discussion on taxonomy and zoogeography. Journal of East African Natural History 101(2):127-222.

Janicki J, Narula N, Ziegler M, Guénard B, Economo EP. 2016. Visualizing and interacting with large-volume biodiversity data using client-server web-mapping applications: the design and implementation of antmaps.org. Ecological Informatics 32:185-193.

Mayr G. 1904. Formiciden aus Ägypten und dem Sudan. In: Jägerskiöld, L. A. 1904. Results of the Swedish Zoological Expedition to Egypt and the White Nile, 1901. Part 1 (no. 9). Uppsala: Library of the Royal University of Uppsala, 11 pp.

Santschi F. 1915a. Nouvelles fourmis d'Algérie, Tunisie et Syrie. Bulletin de la Société d'Histoire Naturelle de l'Afrique du Nord 6:54-63.

Santschi F. 1915b. Nouvelles fourmis d'Afrique. Annales de la Société Entomologique de France 84:244-282.

Santschi F. 1937. Résultats de la Mission scientifique suisse en Angola (2me voyage) 19321933. Fourmis angolaises. Revue Suisse de Zoologie 44:211-250.

Sarnat EM, Economo EP. 2012. The ants of Fiji. University of California Publications in Entomology 132:1-384.

Schlick-Steiner BC, Steiner FM, Seifert B, Stauffer C, Christian E, Crozier RH. 2010. Integrative taxonomy: a multisource approach to exploring biodiversity. Annual Review of Entomology 55: 21-438.

Sharaf MR. 2006. Taxonomic and Ecological Studies on Family Formicidae (Order: Hymenoptera) in Egypt including some Protectorates with a Study of some Insect Fauna associated with Ant Species. Ain Shams University, Faculty of Science, Entomology Department, Cairo, 340 pp. [unpublished thesis].

Sharaf MR, Aldawood AS. 2012. A new ant species of the genus Tetramorium Mayr, 1855 (Hymenoptera, Formicidae) from Saudi Arabia, including a revised key to the Arabian species. PLoS ONE 7 (2), e30811. http://dx.doi.org/10.1371/journal.pone.0030811

Sharaf MR, Aldawood AS. 2013. First occurrence of the Monomorium hildebrandti-group (Hymenoptera: Formicidae), in the Arabian Peninsula, with description of a new species 
772

773

774

775

776

777

778

779

780

781

782

783

784

785

786

787

788

789

790

791

792

793

794

795

796

797

798

799

800

801

802

M. kondratieffi n. sp. Proceedings of the Entomological Society of Washington 115 (1): 75-84.

Sharaf MR, Aldawood AS, El-Hawagry MS. 2012a. A new ant species of the genus Tapinoma (Hymenoptera, Formicidae) from Saudi Arabia with a key to the Arabian species. ZooKeys 212: 35-43. http://dx.doi.org/10.3897/zookeys.212.3325

Sharaf MR, Aldawood AS, El-Hawagry MS. 2012b. First record of the ant subfamily Aenictinae (Hymenoptera, Formicidae) from Saudi Arabia, with the description of a new species. ZooKeys 228: 39-49. http://dx.doi.org/10.3897/zookeys.228.3559

Sharaf MR, Collingwood CA, Al Dhafer HM, Al mutairi MS, Aldawood AS. 2015. New synonyms of two Arabian ants of the genus Monomorium Mayr, 1855 (Hymenoptera, Formicidae). ZooKeys 505: 51-58.

Sharaf MR, Fisher BL, Collingwood CA, Aldawood SA. 2017. Ant fauna (Hymenoptera: Formicidae) of the Socotra Archipelago (Yemen): zoogeography, distribution and description of a new species. Journal of Natural History 51(5-6):317-378.

Terayama M. 2009. A synopsis of the family Formicidae of Taiwan (Insecta: Hymenoptera). Research Bulletin of Kanto Gakuen University. Liberal Arts 17:81-266.

Wheeler W. M. 1922. Ants of the American Museum Congo expedition. New York: Bulletin of the American Museum of Natural History 1139.

Zohary M. 1973. Geobotanical foundations of the Middle East. Vols. 1-2. G. Fischer, Stuttgart, Swets \& Zeitlinger, Amsterdam, 738 pp.

\section{Figure Legends}

Figure 1. Body profile of M. exiguum Forel (CASENT0922302, from www.AntWeb.org

- Michele Esposito) illustrating the measurements used in this paper.

Figure 2. Body in dorsal view of M. exiguum Forel (CASENT0922344, from www.AntWeb.org

- Michele Esposito) illustrating the measurements used in this paper.

Figure 3. Head in full-face view of M. holothir Bolton (CASENT0906392, from www.AntWeb.org

-Estella Ortega) illustrating the measurements used in this paper. 
803 Figure 4. Body in profile showing eyes, pilosity and sculpture on mesosoma and waist segments.

804 A. M. sarawatense (CASENT0280971, from www.AntWeb.org, photographer: Estella Ortega).

805 B. M. holothir (CASENT0906392, from www.AntWeb.org, photographer: Estella Ortega). C. $M$. 806 aeyade (CASENT0922329, from www.AntWeb.org, photographer: Michele Esposito). D. $M$. 807 mohammedi (CASENT0922351, www.AntWeb.org, photographer: Michele Esposito). E. $M$. 808 clavicorne (CASENT0823774, photographer: Francisco Hita Garcia). F. M. exiguum

809 (CASENT0217367, www.AntWeb.org, photographer: Erin Prado).

810 Figure 5. Monomorium aeyade (CASENT0922329,

811 from www.AntWeb.org, photographer: Michele Esposito). A Body in profile. B Body in dorsal

812 view. C Head in full-face view.

813 Figure 6. Monomorium clavicorne (CASENT0823774, photographer: Francisco Hita Garcia). A 814 Body in profile. B Body in dorsal view. C Head in full-face view.

815 Figure 7. Monomorium exiguum (CASENT0922344, from www.AntWeb.org, photographer:

816 Michele Esposito). A Body in profile. B Body in dorsal view. C Head in full-face view.

817 Figure 8. Monomorium holothir (CASENT0906392, from www.AntWeb.org, photographer:

818 Estella Ortega). A Body in profile. B Body in dorsal view. C Head in full-face view.

819 Figure 9. Monomorium mohammedi sp. n. (CASENT0922351, from www.AntWeb.org,

820 photographer: Michele Esposito). A Body in profile. B Body in dorsal view. C Head in full-face 821 view.

822 Figure 10. Monomorium sarawatense (CASENT0280971, from www.AntWeb.org,

823 photographer: Estella Ortega). A Body in profile. B Body in dorsal view. C Head in full-face 824 view.

825 Figure 11. Distribution maps showing the known distribution ranges of the treated species on the 826 Arabian Peninsula, except for $M$. aeyade Collingwood \& Agosti, for which no exact locality data 827 exists A Distribution range of $M$. exiguum Forel (red circles). B Distribution ranges of $M$. 828 clavicorne André (red triangles), M. holothir Bolton (white squares), M. mohammedi sp. n. 829 (green circles), and M. sarawatense Aldawood \& Sharaf (yellow circles), AE: United Arab 830 Emirates, BA: Bahrain, KW: Kuwait, OM: Oman, QA: Qatar, SA: Saudi Arabia, YE: Yemen. 831

832 List of tables: 
833 Table 1. List of species with known distribution ranges. Data extracted from Antmaps

834 (http://antmaps.org; Janicki et al. 2016)

835

836 


\section{Figure 1}

Body profile of M. exiguum Forel (CASENT0922302, from www.AntWeb.org - Michele Esposito) illustrating the used measurements.

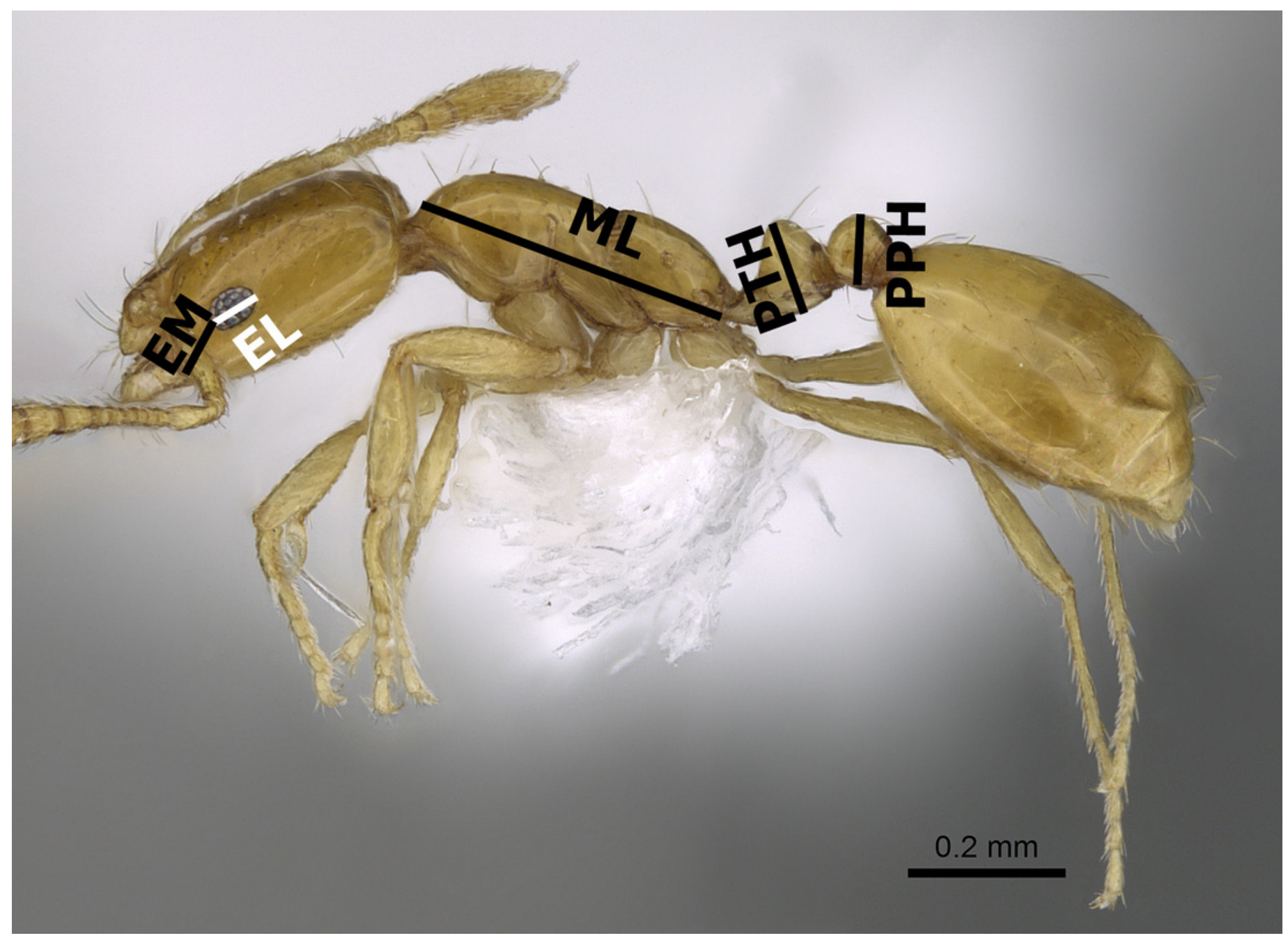




\section{Figure 2}

Body in dorsal view of M. exiguum Forel (CASENT0922344, from www.AntWeb.org Michele Esposito) illustrating the used measurements.

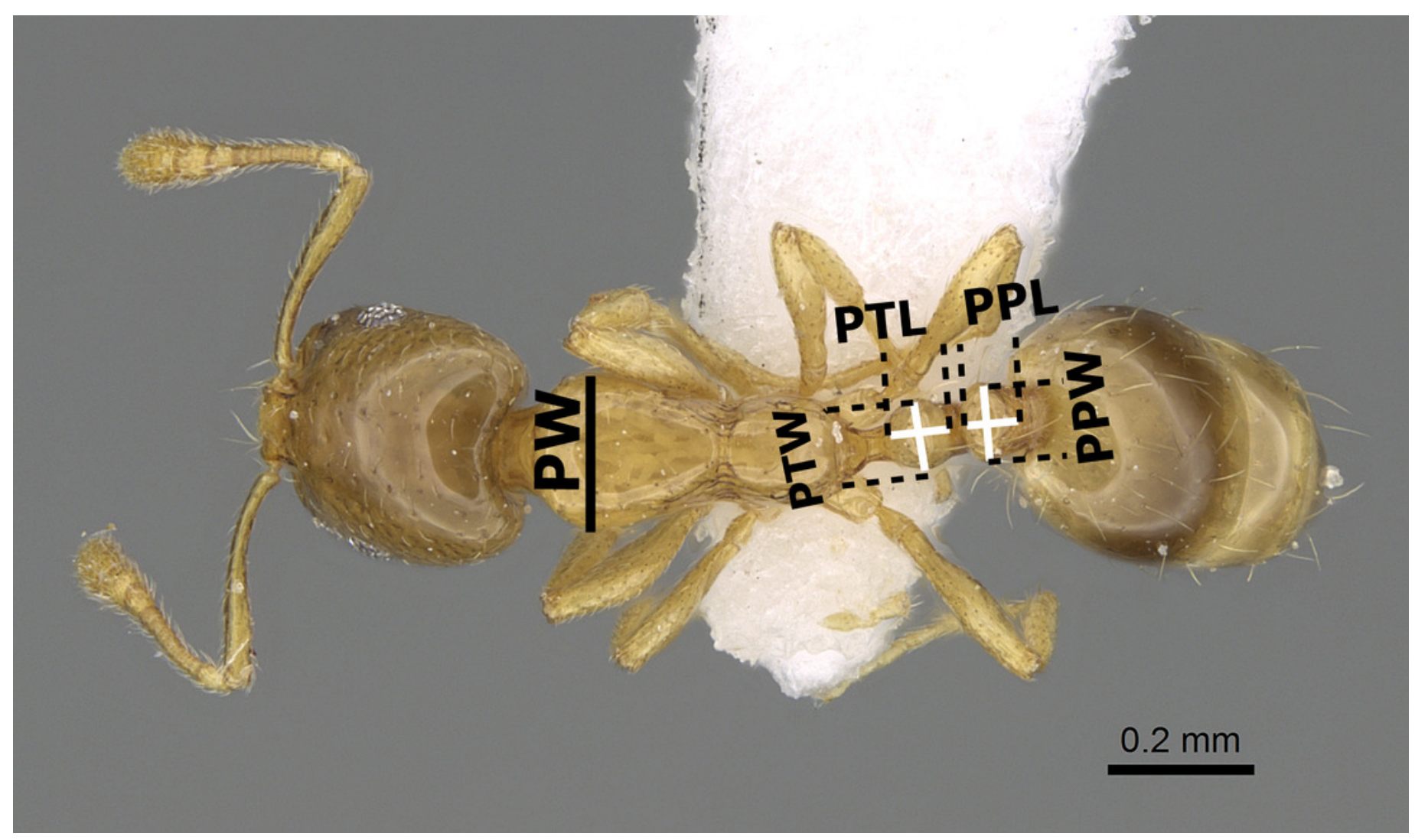




\section{Figure 3}

Head in full-face view of M. holothir Bolton (CASENT0906392, from www.AntWeb.org Estella Ortega) illustrating the used measurements.

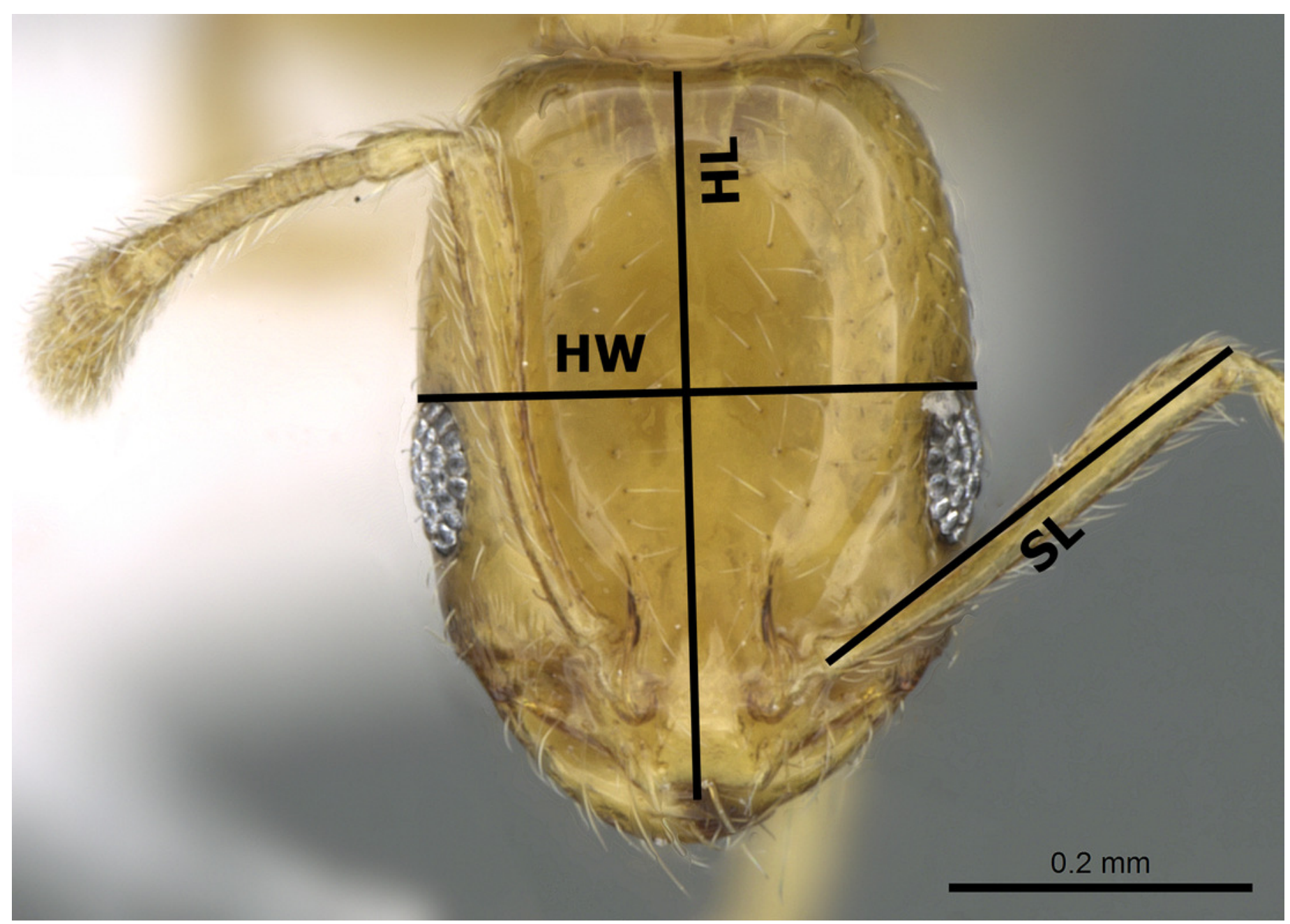




\section{Figure 4}

Body in profile showing eyes, pilosity and sculpture on mesosoma and waist segments.

A. M. sarawatense (CASENT0280971, from www.AntWeb.org, photographer: Estella Ortega).

B. M. holothir (CASENT0906392, from www.AntWeb.org, photographer: Estella Ortega). C. M. aeyade (CASENT0922329, from www.AntWeb.org, photographer: Michele Esposito). D. M. mohammedi (CASENT0922351, www.AntWeb.org, photographer:Michele Esposito). E. M. clavicorne (CASENT0823774, photographer: Francisco Hita Garcia). F. M. exiguum (CASENT0217367, www.AntWeb.org, photographer: Erin Prado). 


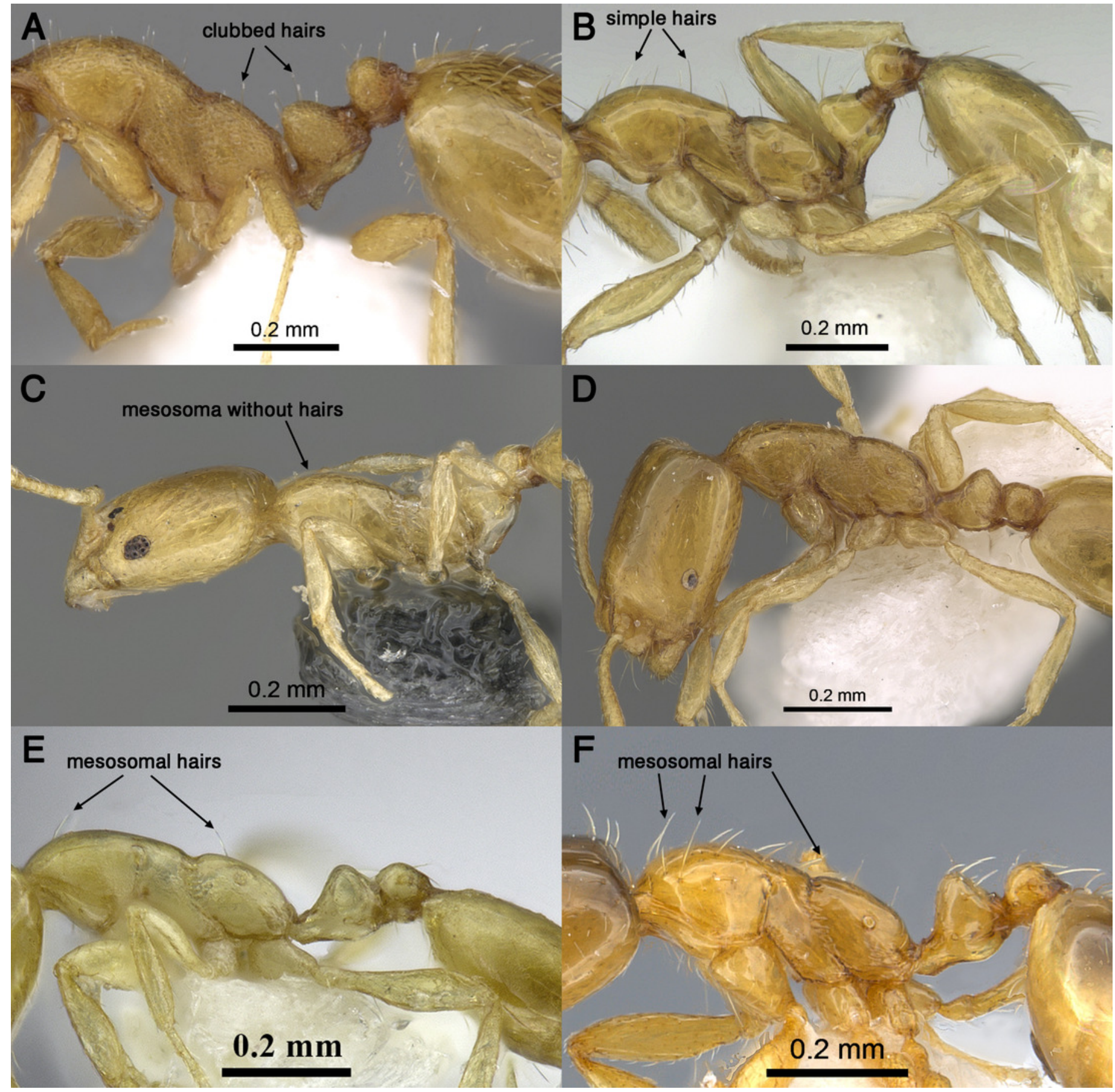


Figure 5

Monomorium aeyade (CASENT0922329, from www.AntWeb.org, photographer: Michele Esposito).

A Body in profile. B Body in dorsal view. C Head in full-face view. 

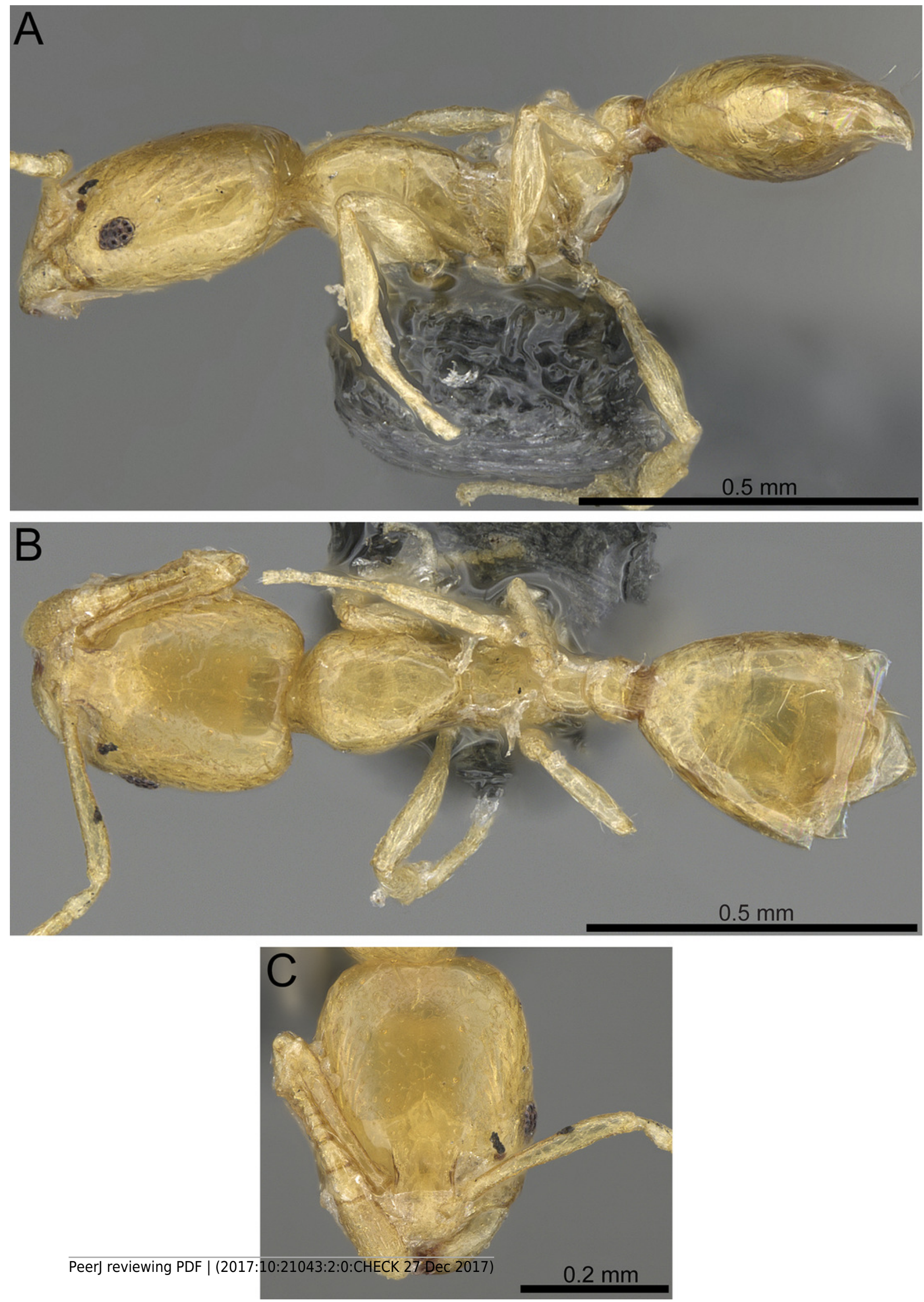
Figure 6

Monomorium clavicorne (CASENT0823774, photographer: Francisco Hita Garcia).

A Body in profile. B Body in dorsal view. C Head in full-face view. 

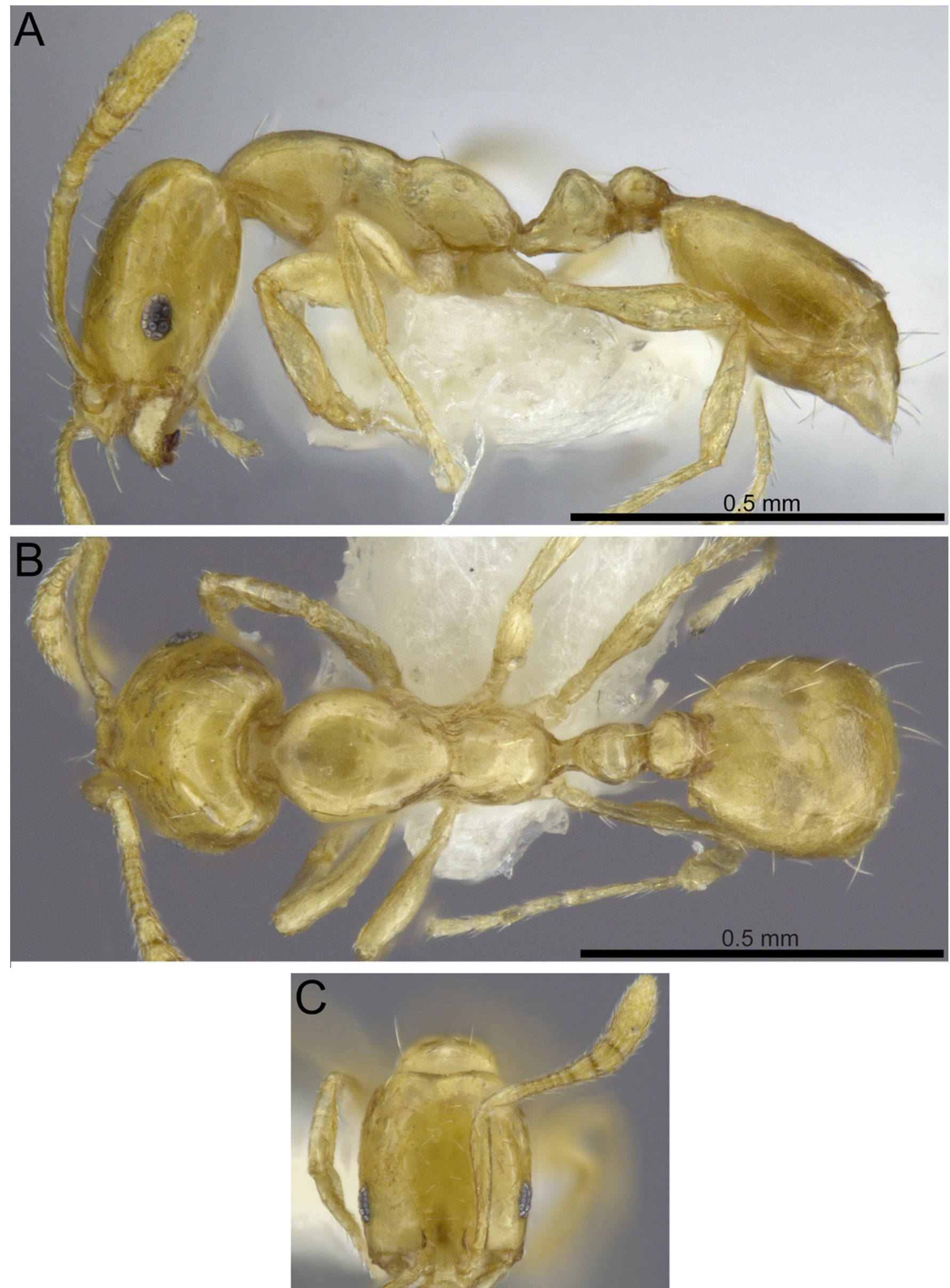
Figure 7

Monomorium exiguum (CASENT0922344, from www.AntWeb.org , photographer: Michele Esposito).

A Body in profile. B Body in dorsal view. C Head in full-face view. 

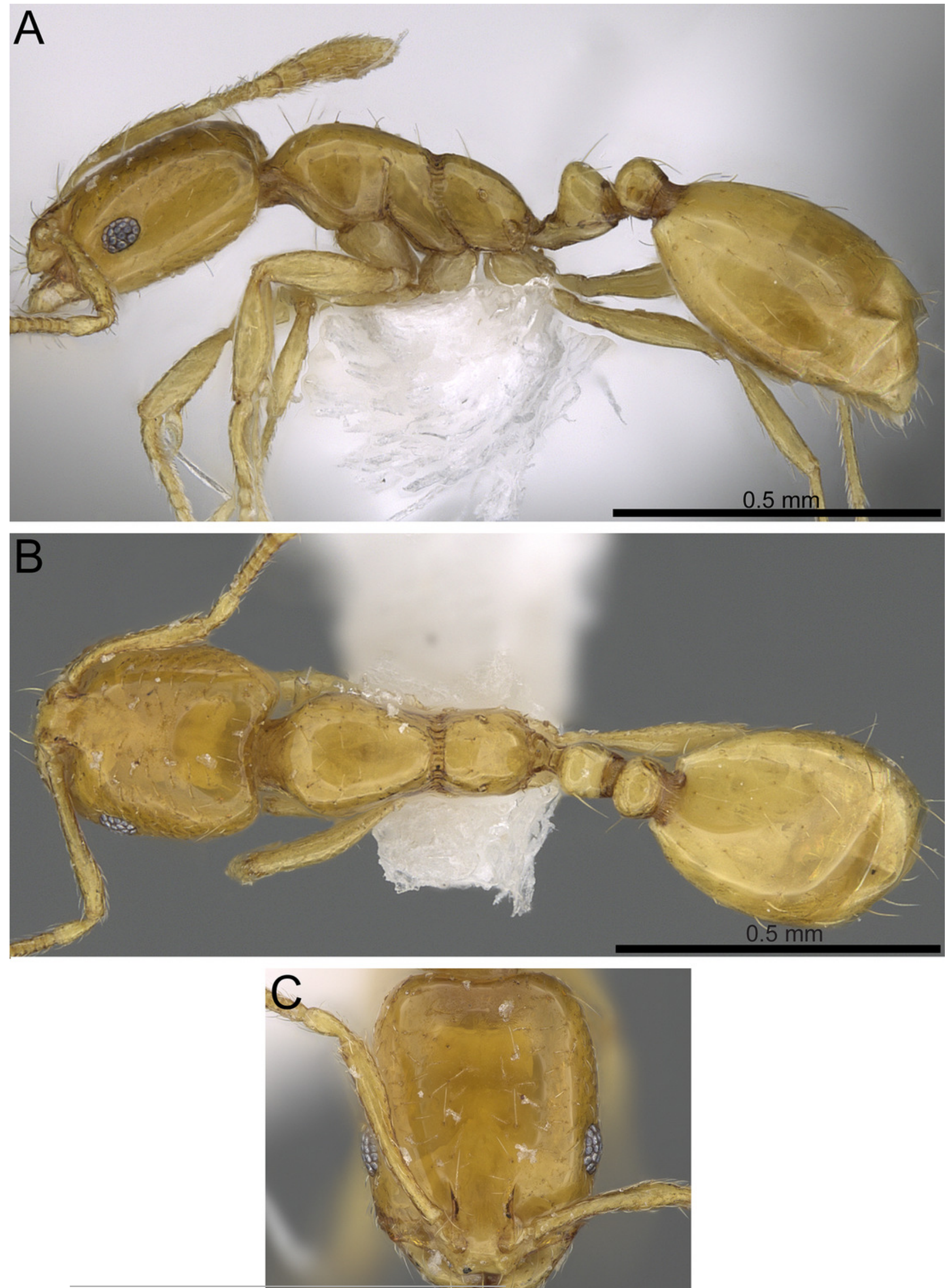
Figure 8

Monomorium holothir (CASENT0906392, from www.AntWeb.org , photographer: Estella Ortega).

A Body in profile. B Body in dorsal view. C Head in full-face view. 

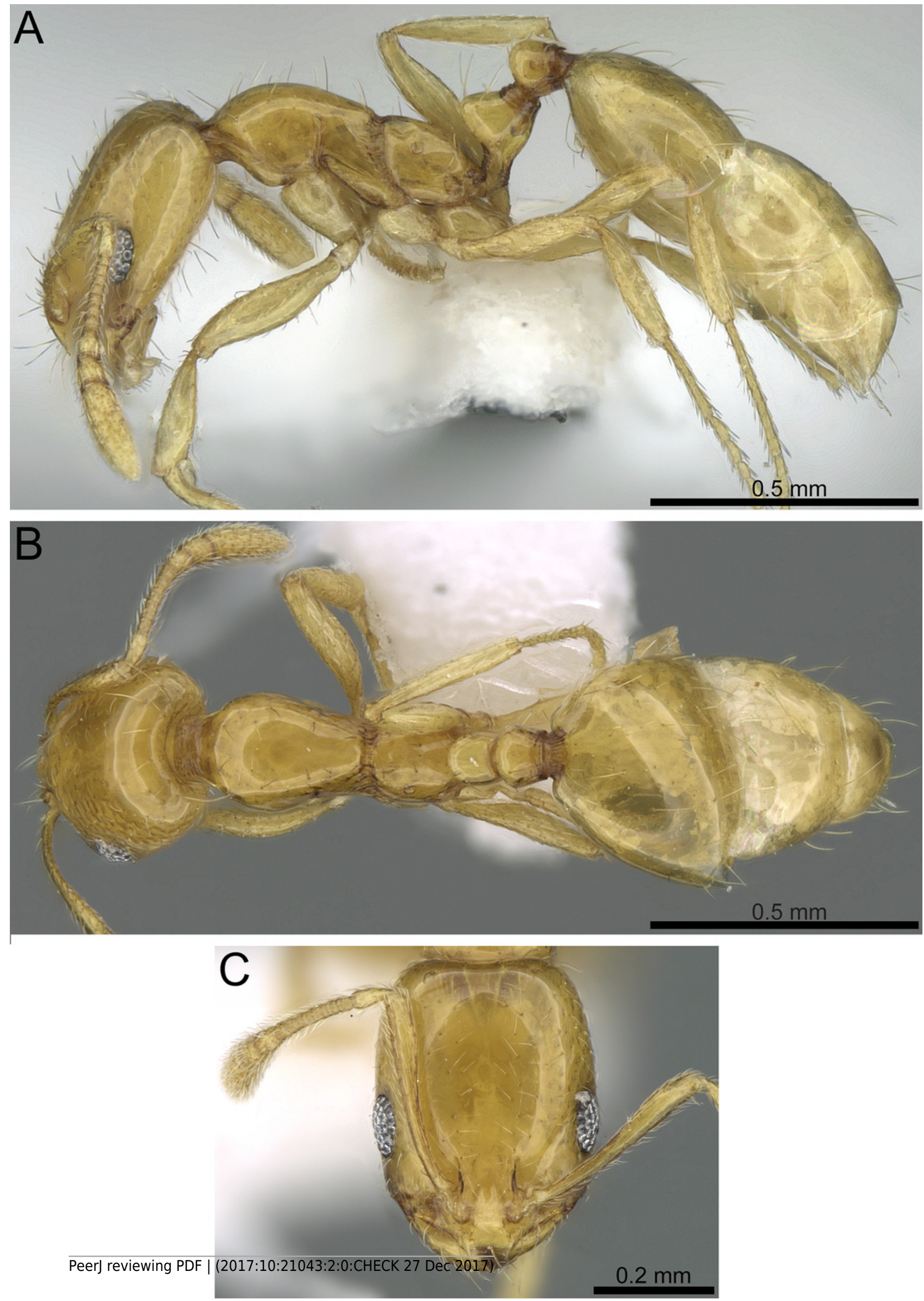
Figure 9

Monomorium mohammedi sp. n. (CASENT0922351, from www.AntWeb.org , photographer: Michele Esposito).

A Body in profile. B Body in dorsal view. C Head in full-face view. 

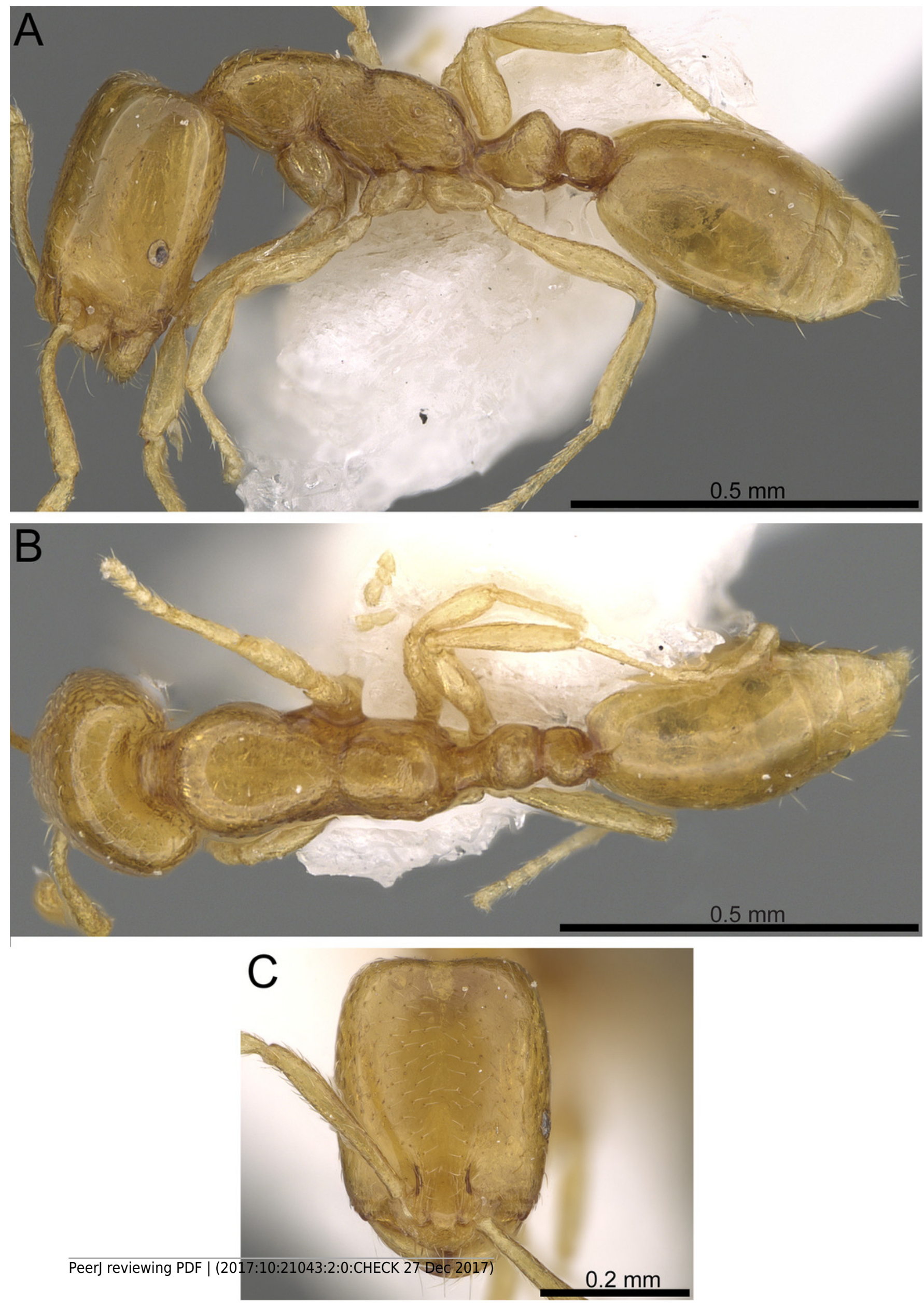
Figure 10

Monomorium sarawatense (CASENT0280971, from www.AntWeb.org , photographer: Estella Ortega).

A Body in profile. B Body in dorsal view. C Head in full-face view. 

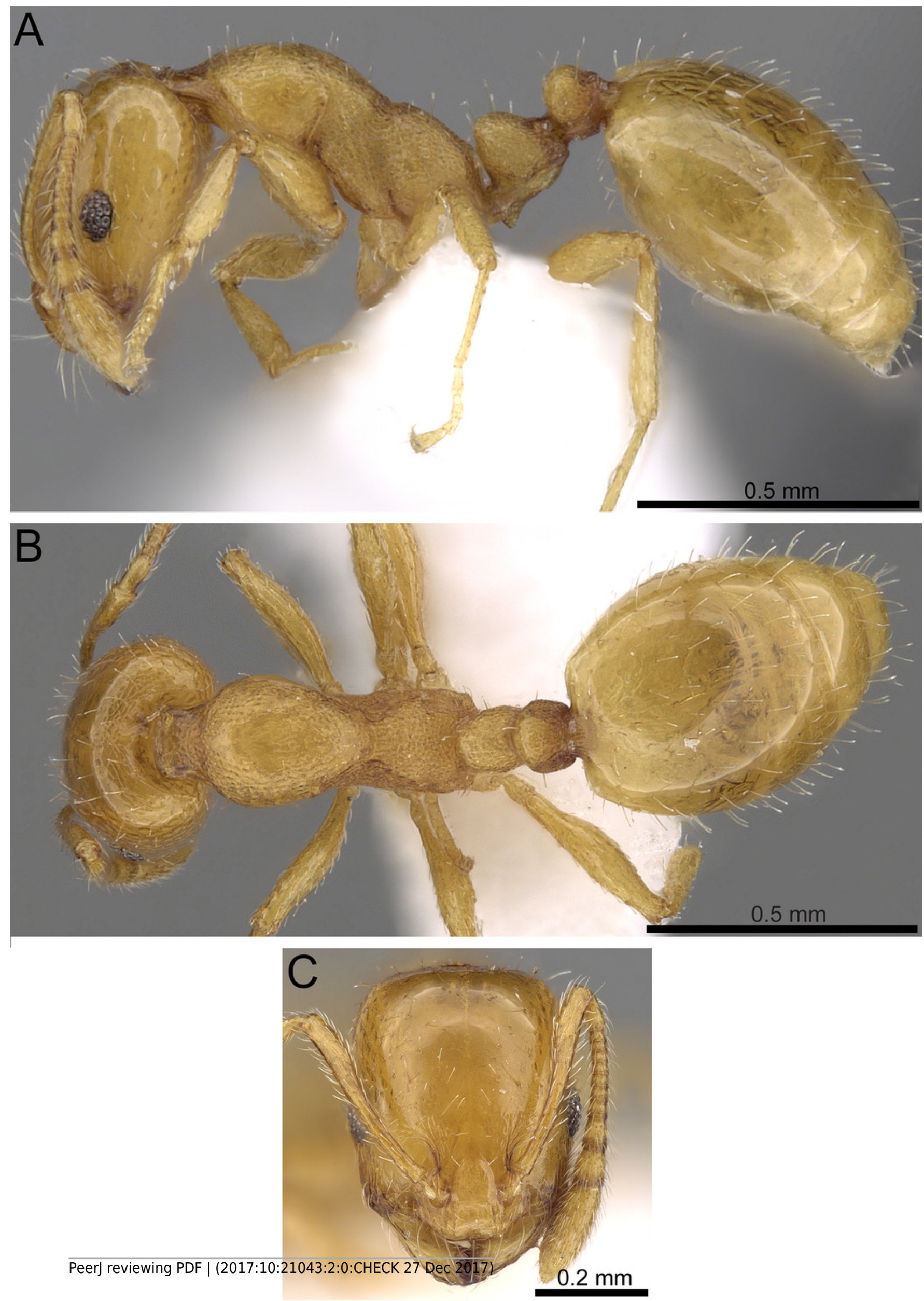


\section{Figure 11}

Distribution maps showing the known distribution ranges of the treated species on the Arabian Peninsula, except for $M$. aeyade Collingwood \& Agosti, for which no exact locality data exists.

A Distribution range of $M$. exiguum Forel (red circles). B Distribution ranges of M. clavicorne André (red triangles), M. holothir Bolton (white squares), M. mohammedi sp. n. (green circles), and M. sarawatense Aldawood \& Sharaf (yellow circles), AE: United Arab Emirates, BA: Bahrain, KW: Kuwait, OM: Oman, QA: Qatar, SA: Saudi Arabia, YE: Yemen. 

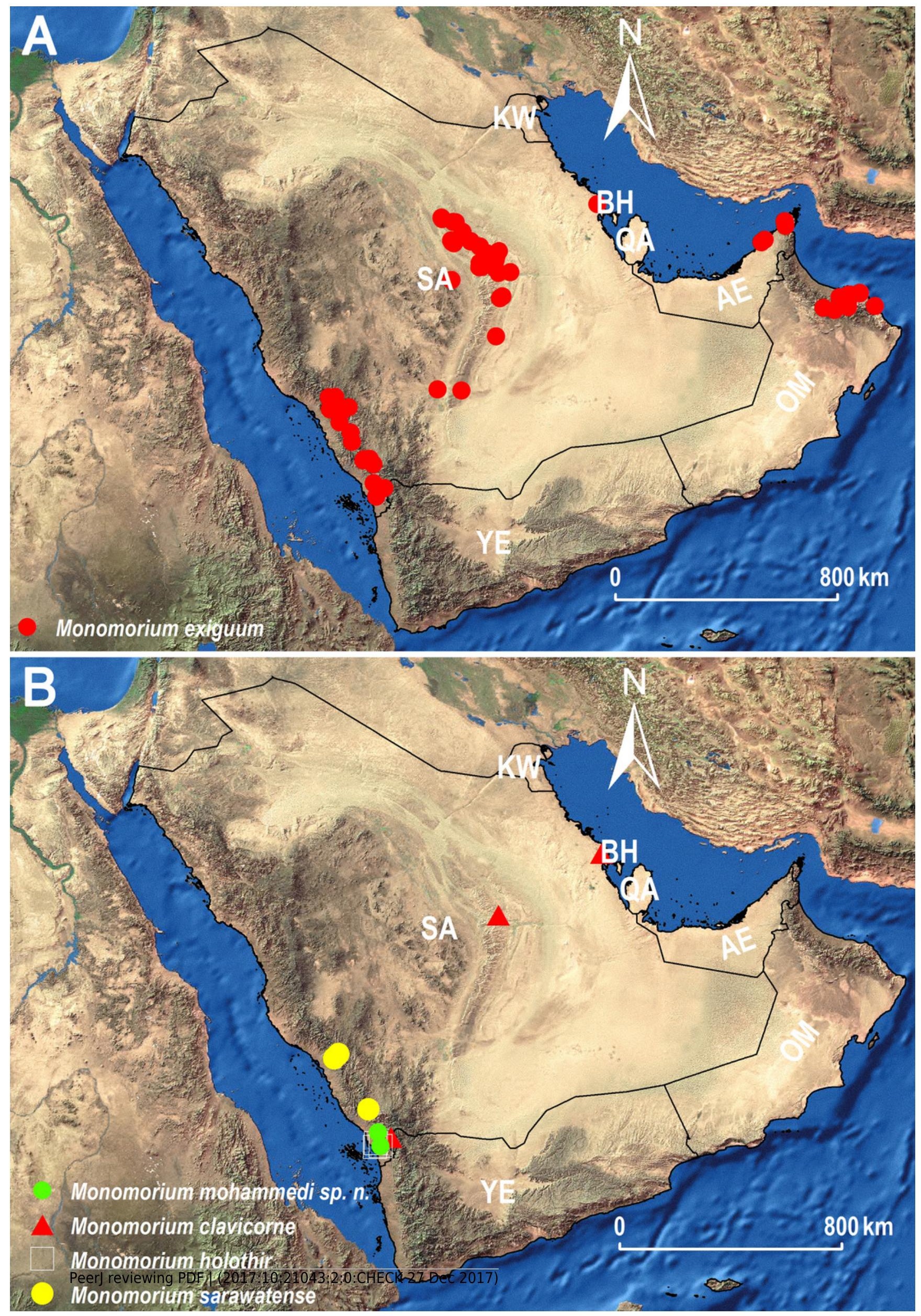


\section{Table $\mathbf{1}$ (on next page)}

List of species with known distribution ranges. Data extracted from Antmaps (http://antmaps.org; Janicki et al. 2016). 


\begin{tabular}{|l|l|}
\hline Species & Distribution \\
\hline M. aeyade & Oman \\
\hline M. clavicorne & $\begin{array}{l}\text { Egypt, Iran, Israel \& Palestine, KSA, Lebanon, Morocco, Sudan, Syria, Tunisia, } \\
\text { Turkey, United Arab Emirates }\end{array}$ \\
\hline $\begin{array}{l}\text { Angola, Cameroon, Cape Verde, Central African Republic, Democratic Republic } \\
\text { of Congo, Ethiopia, Gabon, Ghana, Guinea, Ivory Coast, Kenya, Iran, KSA, } \\
\text { Madagascar, Mozambique, Nigeria, Oman, Spain, South Africa, Tanzania, } \\
\text { Uganda, United Arab Emirates, Yemen, Zimbabwe }\end{array}$ \\
\hline M. holothir & Kenya, KSA \\
\hline M. mohammedi & KSA \\
\hline M. sarawatense & KSA \\
\hline
\end{tabular}

